\title{
Coulomb-assisted $\Sigma^{-}$-nucleus bound states in the $\left(K^{-}, \pi^{+}\right)$reaction
}

\author{
Toru Harada ${ }^{1}$ and Yoshiharu Hirabayashi ${ }^{2}$ \\ ${ }^{1}$ Research Center for Physics and Mathematics, \\ Osaka Electro-Communication University, \\ Neyagawa, Osaka, 572-8530, Japan \\ ${ }^{2}$ Information Initiative Center, Hokkaido University, Sapporo, 060-0811, Japan
}

(Dated: November 1, 2018)

\begin{abstract}
We study a production of Coulomb-assisted $\Sigma^{-}$-nucleus bound states by nuclear $\left(K^{-}, \pi^{+}\right)$reactions within a distorted-wave impulse approximation, so as to examine several types of the $\Sigma$-nucleus potentials that are consistent with the available $\Sigma^{-}$atomic X-ray data and nuclear $\left(\pi^{-}, K^{+}\right)$data. We theoretically demonstrate the inclusive $\left(K^{-}, \pi^{+}\right)$spectra of the $\Sigma^{-}$unstable bound states on ${ }^{28} \mathrm{Si},{ }^{58} \mathrm{Ni}$, and ${ }^{208} \mathrm{~Pb}$ targets at incident $K^{-}$lab momenta $p_{K}=400-800 \mathrm{MeV} / \mathrm{c}$. The results show that the near-recoilless $\left(K^{-}, \pi^{+}\right)$reaction on the ${ }^{58} \mathrm{Ni}$ target gives a clear candidate to confirm properties of the $\Sigma$-nucleus potentials having a repulsion inside the nuclear surface and an attraction outside the nucleus with a sizable absorption, whereas details of the repulsion of the potential at the nuclear center cannot be determined by the inclusive spectra. This is a promising attempt to extract properties of the $\Sigma$-nucleus potential in the nucleus at forthcoming J-PARC experiments, as a full complement to the analyses of the $\Sigma^{-}$atomic and $\left(\pi^{-}, K^{+}\right)$data.

PACS numbers: 21.80.+a, 24.10.Eq, 24.50.+g, 25.80.Pw
\end{abstract}

Keywords: Hypernuclei, Sigma-nucleus potential, DWIA, Sigma-atoms, Bound state 


\section{INTRODUCTION}

It has been discussed that a study of a negatively charged $\Sigma^{-}$hyperon in nuclei would provide valuable information concerning the maximal mass of neutron stars [1], in which a baryon fraction is found to depend on properties of hypernuclear potentials in neutron stars. The $\Sigma^{-}$hyperon in nuclei undergoes a fast decay via strong $\Sigma N \rightarrow \Lambda N$ conversion processes due to the mass difference of $m_{\Sigma}-m_{\Lambda} \simeq 80 \mathrm{MeV}$. Gal and Dover [2] estimated a broad $\Sigma^{-}$ width of $\Gamma_{\Sigma} \simeq 23 \mathrm{MeV}$ in nuclear matter by effectively describing the conversion processes as the imaginary part of a $\Sigma$-nucleus (optical) potential. One of the most important subjects in this research field is to clarify properties of the real and imaginary parts of the $\Sigma$-nucleus potential.

The latest analyses of strong-interaction shifts and widths in $\Sigma^{-}$atoms, which are obtained from the $\Sigma^{-}$atomic X-ray data, have suggested that the $\Sigma$-nucleus potential has a repulsion inside the nucleus and a shallow attraction outside the nuclear surface [3]. However, it should be noticed that the $\Sigma^{-}$atomic energies and widths are not so sensitive to a radial distribution of the $\Sigma$-nucleus potential inside the nucleus.

Noumi and his collaborators [4,5] have performed measurements of $\Sigma$-hypernuclear production by inclusive $\left(\pi^{-}, K^{+}\right)$reactions on $\mathrm{C}$, Si, Ni, In and Bi targets at $p_{\pi}=1.20 \mathrm{GeV} / \mathrm{c}$ in KEK-E438 experiments. Several theoretical analyses have also suggested that a repulsive component in the $\Sigma$-nucleus potentials is needed to reproduce the observed spectra of $\left(\pi^{-}, K^{+}\right)$reactions on nuclear targets [5-7]. This repulsion originates from the $\Sigma N$ $T=3 / 2,{ }^{3} S_{1}$ channel, of which state corresponds to a quark Pauli-forbidden state in the baryon-baryon system [8,9], and it is a candidate for the appearance of quark degrees of freedom in nuclear physics.

In a previous paper [7], we have succeeded to explain the ${ }^{28} \mathrm{Si}\left(\pi^{-}, K^{+}\right)$data as well as the $\Sigma^{-}$atomic X-ray data simultaneously, by using the $\Sigma$-nucleus potentials that have a repulsion inside the nuclear surface and an attraction outside the nucleus with a sizable absorption [10]. Nevertheless, the radial distribution of the potential inside the nucleus and its strength at the center are hardly determined by fits to the $\Sigma^{-}$QF spectrum $[7,11]$. Since the $\Sigma^{-}$continuum states in the QF region are favored in the $\left(\pi^{-}, K^{+}\right)$reaction due to its large momentum transfer of $\sim 400 \mathrm{MeV} / \mathrm{c}$, quantitative ambiguity of the potential cannot be resolved in the analysis of the complicated continuum states over a wide excitation-energy 
range [7].

One expects that there are the $\Sigma^{-}$-nucleus bound states assisted by the Coulomb attraction even if the $\Sigma$-nucleus potential is repulsive. The wave functions of the $\Sigma^{-}$states are sizably moderated by strong interactions because a rms radius for a $\Sigma^{-}$atomic $1 s$ state in medium-to-heavy nuclei is comparable to its nuclear size, e.g., $\left\langle r^{2}\right\rangle_{1 s}^{1 / 2}=4.2 \mathrm{fm}$ for a ${ }^{57}$ Co core-nucleus where $R=1.1 A^{1 / 3}=4.23 \mathrm{fm}$. Yamazaki et al. [12] called these states "Coulomb-assisted hybrid bound states", rather than $\Sigma^{-}$atomic states. Can we clearly observe such a $\Sigma^{-}$bound state?

In this paper, we theoretically demonstrate a production of the Coulomb-assisted $\Sigma^{-}$nucleus bound states by nuclear $\left(K^{-}, \pi^{+}\right)$reactions for forthcoming J-PARC experiments. It is well known that the nuclear $\left(K^{-}, \pi^{+}\right)$reaction provides the recoilless condition for a $\Sigma^{-}$production (see Fig. 1), which leads to the optimum population of a $\Delta L=0$ transition on nuclear targets [13]. For medium-to-heavy nuclei, however, it seems that individual $\Sigma^{-}$levels with broad widths are unseparated because the level densities are high in the $\Sigma^{-}$ bound region. Thus an appropriate momentum transfer near recoilless conditions is required to selectively populate a non-substitutional bound state in $\Sigma^{-}$-nucleus systems. We perform a calculation of the inclusive spectra on ${ }^{28} \mathrm{Si},{ }^{58} \mathrm{Ni}$ and ${ }^{208} \mathrm{~Pb}$ targets within a distorted-wave impulse approximation (DWIA) in order to examine properties of the $\Sigma$-nucleus potentials. We attempt to extract quantitative information on the repulsive component of the $\Sigma$-nucleus potentials from the calculated $\left(K^{-}, \pi^{+}\right)$spectra. This is a natural extension of examinations of the $\Sigma$-nucleus potentials by $\Sigma^{-}$production reactions on nuclear targets $[7,11,14]$.

The outline of this paper is as follows: In Sect. II, we briefly mention a framework for the nuclear $\left(K^{-}, \pi^{+}\right)$reactions in a DWIA. In Sect. III, we show properties of several $\Sigma$-nucleus potentials that are consistent with the $\Sigma^{-}$atomic X-ray data [3] and nuclear $\left(\pi^{-}, K^{+}\right)$data $[4,5]$. In Sect. IV, we calculate the binding energies and widths of the Coulomb-assisted $\Sigma^{-}$-nucleus bound states for $\Sigma^{-}-^{27} \mathrm{Al}, \Sigma^{-}-57 \mathrm{Co}$ and $\Sigma^{-}-207 \mathrm{Tl}$ systems. In Sect. V, we show numerical results of the inclusive spectra in the $\Sigma^{-}$bound region by the $\left(K^{-}, \pi^{+}\right)$reactions on ${ }^{28} \mathrm{Si},{ }^{58} \mathrm{Ni}$, and ${ }^{208} \mathrm{~Pb}$ targets at $p_{K}=400-800 \mathrm{MeV} / \mathrm{c}$ and $\theta_{\text {lab }}=5^{\circ}$. We discuss the dependence of a peak structure in the spectra on various types of the $\Sigma$-nucleus potentials in order to discriminate among these potentials. Summary and conclusion are given in Sect. VI. 


\section{THEORY}

\section{A. Distorted-wave impulse approximation (DWIA)}

Hypernuclear production cross sections have been usually calculated with the framework of a DWIA [15-20]. The double-differential cross section for the $\left(K^{-}, \pi^{+}\right)$reaction at a forward-direction angle $\theta_{\text {lab }}$ in a lab frame is written $[21,22]$ by

$$
\frac{d^{2} \sigma}{d E_{\pi} d \Omega_{\pi}}=\beta \frac{1}{\left[J_{A}\right]} \sum_{M_{A}} \sum_{B, M_{B}}\left|\left\langle\Psi_{B}|\hat{F}| \Psi_{A}\right\rangle\right|^{2} \delta\left(\omega+E_{\pi}-E_{K}\right)
$$

with

$$
\hat{F}=\int d \boldsymbol{r} \chi_{\boldsymbol{p}_{\pi}}^{(-) *}(\boldsymbol{r}) \chi_{\boldsymbol{p}_{K}}^{(+)}(\boldsymbol{r}) \sum_{j=1}^{A}\left(\bar{f}+i \bar{g} \boldsymbol{\sigma}_{j} \cdot \hat{\boldsymbol{n}}\right) \hat{\mathcal{O}}_{j} \delta\left(\boldsymbol{r}-\boldsymbol{r}_{j}\right),
$$

where $\left|\Psi_{B}\right\rangle$ is a final state of the $\Sigma^{-}$nuclear system with a total spin $J_{B}$, and $\left|\Psi_{A}\right\rangle$ is an initial state of the target nucleus with a total spin $J_{A}$. The momentum and energy transfer to the $\Sigma^{-}$final state is given by

$$
\boldsymbol{q}_{\Sigma}=\boldsymbol{p}_{K}-\boldsymbol{p}_{\pi}, \quad \omega=E_{K}-E_{\pi},
$$

where $\boldsymbol{p}_{K}$ and $\boldsymbol{p}_{\pi}\left(E_{K}\right.$ and $\left.E_{\pi}\right)$ are the lab momenta (energies) of the incident $K^{-}$and outgoing $\pi^{+}$in the many-body $K^{-}+{ }^{A} \mathrm{Z} \rightarrow \pi^{+}+{ }_{\Sigma^{-}}^{A}(\mathrm{Z}-2)^{*}$ reaction, respectively. The kinematical factor $\beta[23,24]$ expresses the translation from the two-body $K^{-}{ }^{-p}$ lab system to the $K^{-}-{ }^{A} \mathrm{Z}$ lab system [19], which is given by

$$
\beta=\left(1+\frac{E_{\pi}^{(0)}}{E_{\Sigma}^{(0)}} \frac{p_{\pi}^{(0)}-p_{K}^{(0)} \cos \theta_{\mathrm{lab}}}{p_{\pi}^{(0)}}\right) \frac{p_{\pi} E_{\pi}}{p_{\pi}^{(0)} E_{\pi}^{(0)}},
$$

where $p_{K}^{(0)}$ and $p_{\pi}^{(0)}\left(E_{\pi}^{(0)}\right.$ and $\left.E_{\Sigma}^{(0)}\right)$ are the momenta of $K^{-}$and $\pi^{+}$(energies of $\pi^{+}$and $\Sigma^{-}$) in the two-body $K^{-}+p \rightarrow \pi^{+}+\Sigma^{-}$reaction, respectively. $\bar{f}$ and $\bar{g}$ in Eq. (2) denote the non-spin-flip and spin-flip lab amplitudes, respectively, for the $K^{-}+p \rightarrow \pi^{+}+\Sigma^{-}$ elementary process in nuclear medium, and the operator $\hat{\mathcal{O}}_{j}$ changes a $j$ th nucleon to the $\Sigma^{-}$in the nucleus, $\hat{\mathcal{O}}_{j}\left|p_{j}\right\rangle=\left|\Sigma^{-}\right\rangle . \chi_{\boldsymbol{p}_{\pi}}^{(-)}$and $\chi_{\boldsymbol{p}_{K}}^{(+)}$are the distorted waves for the outgoing $\pi^{+}$and incoming $K^{-}$, respectively. The computational procedure for the distorted waves is simplified with the help of the eikonal approximation [16, 18]. The meson distorted waves are expressed $[25]$ as

$$
\chi_{\boldsymbol{p}_{\pi}}^{(-) *}(\boldsymbol{r}) \chi_{\boldsymbol{p}_{K}}^{(+)}(\boldsymbol{r})=\sum_{L} \sqrt{4 \pi(2 L+1)} i^{L} \tilde{j}_{L}(q, r) Y_{L}^{0}(\hat{\boldsymbol{r}}),
$$


where $\tilde{j}_{L}(q, r)$ is a radial distorted wave with the angular-momentum $L$ and momentum transfer $q$. Here we used total cross sections of $\sigma_{K}=32 \mathrm{mb}$ for $K^{-} N$ scattering and $\sigma_{\pi}=30$ mb for $\pi^{+} N$ one, and $\alpha_{K}=\alpha_{\pi}=0$ [18], as distortion parameters in $\tilde{j}_{L}(q, r)$, together with a matter density distribution fitted to the charge radius [26].

\section{TABLE I}

For the nuclear targets, we use single-particle wave functions for a proton, which are calculated with a Woods-Saxon (WS) potential [27]:

$$
U_{N}(r)=V_{0}^{N} f(r)+V_{l s}^{N}(\boldsymbol{l} \cdot \boldsymbol{s}) r_{0}^{2} \frac{1}{r} \frac{d}{d r} f(r)
$$

with $f(r)=[1+\exp ((r-R) / a)]^{-1}$, where $V_{l s}^{N}=-0.44 V_{0}^{N}, a=0.67 \mathrm{fm}, r_{0}=1.27 \mathrm{fm}$ and $R=r_{0} A^{1 / 3}$. We choose the strengths of $V_{0}^{N}=-59.7,-59.0$ and $-61.7 \mathrm{MeV}$ by fits to the charge radii [26] of $\left\langle r_{\mathrm{ch}}^{2}\right\rangle^{1 / 2}=3.09,3.77$ and $5.51 \mathrm{fm}$ for ${ }^{28} \mathrm{Si},{ }^{58} \mathrm{Ni}$ and ${ }^{208} \mathrm{~Pb}$, respectively. Because deep proton-hole states play an important role in describing the $\Sigma^{-}$excited and continuum states [25], we take the single-particle energies and widths from $\left(e, e^{\prime} p\right)$ data for nuclei $[28,29]$. For deep-hole states that are unknown experimentally in ${ }^{58} \mathrm{Ni}$ and ${ }^{208} \mathrm{~Pb}$, we also use the energies obtained by density-dependent Hartree-Fock calculations [30]. In Table I, we list these energies and widths for ${ }^{28} \mathrm{Si},{ }^{58} \mathrm{Ni}$ and ${ }^{208} \mathrm{~Pb}$, which are input into this calculation.

\section{B. Fermi-averaged $K^{-}+p \rightarrow \pi^{+}+\Sigma^{-}$cross section}

When we calculate the nuclear $\left(K^{-}, \pi^{+}\right)$cross sections with the $K^{-}+p \rightarrow \pi^{+}+\Sigma^{-}$ amplitudes, it is important to take into account the Fermi-motion of a struck nucleon with a Fermi momentum $p_{F} \simeq 270 \mathrm{MeV} / \mathrm{c}$ in nuclear medium [31, 32]. This effect is considerably enhanced near narrow $\Lambda / \Sigma$ resonances because their widths are smaller than the Fermi-motion energy of the struck nucleon, e.g., $D_{03}(1520)$ at $p_{K} \simeq 390 \mathrm{MeV} / \mathrm{c}, S_{01}(1670)$, $D_{03}(1690)$ and $D_{13}(1670)$ at $\sim 750 \mathrm{MeV} / \mathrm{c}$, as seen in Fig. 1(a). According to the procedure by Rosental and Tabakin [32], we perform the Fermi-averaging of the $K^{-}+p \rightarrow \pi^{+}+\Sigma^{-}$ scattering $T$-matrix obtained by Gopal et al. [33]. 
In Fig. 1, we show the Fermi-averaged lab cross section of the $K^{-}+p \rightarrow \pi^{+}+\Sigma^{-}$reaction on nuclei,

$$
\left\langle\frac{d \sigma}{d \Omega}\right\rangle_{\text {lab }}^{K^{-} p \rightarrow \pi^{+} \Sigma^{-}}=|\bar{f}|^{2}+|\bar{g}|^{2},
$$

at the detected $\pi^{+}$angles $\theta_{\text {lab }}=0^{\circ}$ and $10^{\circ}$, as a function of the incident $K^{-}$lab momentum $p_{K}$, together with the momentum transfer $q_{\Sigma}$ in the nuclear $\left(K^{-}, \pi^{+}\right)$reaction. $|\bar{f}|^{2}$ and $|\bar{g}|^{2}$ denote the non-spin-flip and spin-flip components of the Fermi-averaged lab cross sections, respectively. No data are measured in the low incident $K^{-}$momentum region below about $300 \mathrm{MeV} / \mathrm{c}$. The shape of the Fermi-averaged cross section near 400-800 MeV/c sizably becomes broader, and its value is not so changed by a choice of the target, as discussed by Dover et al. $[22,34]$. Since the spin-flip cross sections of $|\bar{g}|^{2}$ is negligible, we consider only the non-spin-flip process in the nuclear $\left(K^{-}, \pi^{+}\right)$reaction in this paper.

\section{Green's function technique}

To evaluate the inclusive spectrum in Eq. (1), here we employ the Green's function method $[21,35]$. This technique can describe an unstable hadron nuclear system such as a $\Sigma^{-}, \Xi^{-}$or $K^{-}$nuclear state very well $[7,11,23,25]$. The complete Green's function $G$ provides all information concerning $\Sigma$-nucleus dynamics as a function of the energy transfer $\omega=E_{B}-E_{A}$ or the energy $E$ measured from the $\Sigma^{-}+$core-nucleus threshold,

$$
E=E_{B}-\left(m_{\Sigma^{-}}+M_{C}\right)=-B_{\Sigma^{-}}
$$

where $m_{\Sigma^{-}}$and $M_{C}$ are masses of the $\Sigma^{-}$and the core-nucleus, respectively. It is obtained by solving the following potential problems:

$$
G(E)=G^{(0)}(E)+G^{(0)}(E)\left\{U_{\Sigma}+U_{\text {Coul }}\right\} G(E)
$$

where $G^{(0)}(E)$ is a free Green's function. $U_{\Sigma}$ is the $\Sigma^{-}$-nucleus potential, and $U_{\text {Coul }}$ is the finite Coulomb potential between the $\Sigma^{-}$and the core-nucleus. By a use of the complete Green's function $G$, a sum of the final states $B$ of Eq. (1) is given as

$$
\sum_{B}\left|\Psi_{B}\right\rangle \delta\left(\omega+E_{A}-E_{B}\right)\left\langle\Psi_{B}\right|=-\frac{1}{\pi} \operatorname{Im} G(\omega) .
$$

Thus the inclusive spectrum of the double-differential cross section is rewritten as

$$
\frac{d^{2} \sigma}{d \Omega_{\pi} d E_{\pi}}=\beta\left\langle\frac{d \sigma}{d \Omega}\right\rangle_{\mathrm{lab}}^{K^{-} p \rightarrow \pi^{+} \Sigma^{-}} S(\omega)
$$


with the strength function $S(\omega)$, which is given by

$$
S(\omega)=-\frac{1}{\pi} \operatorname{Im} \sum_{\alpha^{\prime} \alpha} \int d \boldsymbol{r}^{\prime} d \boldsymbol{r} f_{\alpha^{\prime}}^{\dagger}\left(\boldsymbol{r}^{\prime}\right) G_{\alpha^{\prime} \alpha}\left(\omega ; \boldsymbol{r}^{\prime}, \boldsymbol{r}\right) f_{\alpha}(\boldsymbol{r}),
$$

where $\boldsymbol{r}$ is the relative coordinate between the $\Sigma^{-}$and the core-nucleus. $f_{\alpha}(\boldsymbol{r})$ presents the production function via $\Sigma^{-}$-nucleus doorways that are excited initially as

$$
f_{\alpha}(\boldsymbol{r})=\chi_{\boldsymbol{p}_{\pi}}^{(-) *}\left(\frac{M_{C}}{M_{B}} \boldsymbol{r}\right) \chi_{\boldsymbol{p}_{K}}^{(+)}\left(\frac{M_{C}}{M_{A}} \boldsymbol{r}\right)\left\langle\alpha\left|\hat{\psi}_{N}(\boldsymbol{r})\right| \Psi_{A}\right\rangle,
$$

where $\left\langle\alpha\left|\hat{\psi}_{N}(\boldsymbol{r})\right| \Psi_{A}\right\rangle$ is a hole-state wave function for a struck nucleon in the target, and $\alpha$ denotes the complete set of eigenstates for the system. The factors of $M_{C} / M_{B}$ and $M_{C} / M_{A}$ take into account the recoil effects.

Here we consider the $\Sigma^{-}$-nucleus system in a non-relativistic framework. Since $U_{\Sigma}$ has an imaginary part, the Hamiltonian $\mathcal{H}=T+U_{\Sigma}$ is non-Hermitian. Thus the Schrödinger equations are written as

$$
\mathcal{H} \varphi_{n}=E_{n} \varphi_{n}, \quad \mathcal{H}^{\dagger} \tilde{\varphi}_{n}=E_{n}^{*} \tilde{\varphi}_{n},
$$

where $E_{n}$ is a complex eigenvalue. The $\Sigma^{-}$nuclear binding energy and width for a $\Sigma^{-}$ unstable bound state can be evaluated as

$$
E_{n}=\left(k_{n}^{(\text {pole })}\right)^{2} / 2 \mu=-B_{n}-i \Gamma_{n} / 2
$$

where $k_{n}^{(\text {pole })}$ denotes a pole position of the bound state in the complex $k$-plane $\left(\operatorname{Re} k_{n}^{(\text {pole })}<0\right.$, $\left.\operatorname{Im} k_{n}^{(\text {pole })}>0\right)$, and $\mu$ is the reduced mass of the $\Sigma^{-}$-nucleus system. $\varphi_{n}$ is a wave function of the eigenstate labeled by $k_{n}^{(\text {pole) }}$ and $\tilde{\varphi}_{n}$ is the wave function given by a biorthogonal set; its conjugate state becomes $\left(\tilde{\varphi}_{n}\right)^{*}=\varphi_{n}$, of which radial wave functions must be normalized by so-called $c$-products [36],

$$
\begin{aligned}
& \int_{0}^{\infty} r^{2} d r\left(\tilde{\varphi}_{n}(r)\right)^{*} \varphi_{n}(r) \\
& =\int_{0}^{\infty} r^{2} d r\left(\varphi_{n}(r)\right)^{2}=1,
\end{aligned}
$$

under the boundary condition for decaying states [37]. The completeness relation for the complete Green's function is written as

$$
\begin{aligned}
G\left(E ; r^{\prime}, r\right) & =\sum_{n} \frac{\varphi_{n}\left(r^{\prime}\right)\left(\tilde{\varphi}_{n}(r)\right)^{*}}{E-E_{n}+i \epsilon} \\
& +\frac{2}{\pi} \int_{0}^{\infty} d k \frac{k^{2} S(k) u\left(k, r^{\prime}\right)(\tilde{u}(k, r))^{*}}{E-E_{k}+i \epsilon}
\end{aligned}
$$


where the summation over $n$ includes all the pole of the $S$-matrix in the complex $k$-plane, and $u(k, r)$ is a scattering wave function. In the bound region, Green's function might be expanded [35] as

$$
G\left(E ; r^{\prime}, r\right)=\sum_{n} G_{n}^{(\text {pole })}\left(E ; r^{\prime}, r\right)+G^{(\mathrm{bg})}\left(E ; r^{\prime}, r\right)
$$

where the pole contribution of the bound state can be expressed as

$$
G_{n}^{(\mathrm{pole})}\left(E ; r^{\prime}, r\right)=\frac{\varphi_{n}\left(r^{\prime}\right)\left(\tilde{\varphi}_{n}(r)\right)^{*}}{E-E_{n}+i \epsilon}
$$

and $G^{(\mathrm{bg})}\left(E ; r^{\prime}, r\right)$ indicates the background contribution. Note that the interference by the background term or other pole terms occasionally affects the spectrum in the continuum region $E>0$ near the $\Sigma^{-}$threshold if $\left|\operatorname{Im} U_{\Sigma}\right|$ is large [35].

\section{Integrated cross sections and the complex effective number}

To study a structure of hypernuclear bound states, the integrated cross sections of these states have been often evaluated in DWIA calculations [15-20]. The angular distributions of the cross section are presented as a function of $\theta_{\text {lab }}$ or $q_{\Sigma}$. The integrated cross section of the $\Sigma^{-}$unstable bound state with $\left(j_{p}^{-1} j_{\Sigma}\right) J_{B}$ in the nuclear $\left(K^{-}, \pi^{+}\right)$reaction is written as (see Appendix B in Ref. [24]):

$$
\begin{aligned}
\left(\frac{d \sigma}{d \Omega_{\pi}}\right) & =\int d E_{\pi}\left(\frac{d^{2} \sigma}{d E_{\pi} d \Omega_{\pi}}\right) \\
& =\bar{\alpha}\left\langle\frac{d \sigma}{d \Omega}\right\rangle_{\mathrm{lab}}^{K^{-} p \rightarrow \pi^{+} \Sigma^{-}} \operatorname{Re} P_{\mathrm{eff}}^{\left(j_{p}^{-1} j_{\Sigma}\right) J_{B}},
\end{aligned}
$$

where $\bar{\alpha}$ is a kinematical factor $[18-20,23]$ defined by

$$
\bar{\alpha}=\beta\left(1+\frac{E_{\pi}}{E_{B}} \frac{p_{\pi}-p_{K} \cos \theta_{\mathrm{lab}}}{p_{\pi}}\right)^{-1},
$$

and $P_{\text {eff }}$ is the complex effective number of a proton [24], which describes all information on the nuclear structure of the unstable bound systems. When the potential has no imaginary part, $P_{\text {eff }}$ becomes a real number. This approach also provides a good insight into a signal of $\Sigma^{-}$bound states caused by a complex potential $U_{\Sigma}$, as discussed in Refs. $[24,35]$. 
For a closed-shell target with $J^{\pi}=0^{+}$, the complex effective number of a proton for the transition $J_{A} \rightarrow\left(j_{p}^{-1} j_{\Sigma}\right) J_{B}$ is written as

$$
\begin{aligned}
P_{\mathrm{eff}}^{\left(j_{p}^{-1} j_{\Sigma}\right) J_{B}}= & \left(2 J_{B}+1\right)\left(2 j_{\Sigma}+1\right)\left(2 j_{p}+1\right) \\
& \times\left(\begin{array}{ccc}
j_{\Sigma} & j_{p} & J_{B} \\
\frac{1}{2} & -\frac{1}{2} & 0
\end{array}\right) F(q) F^{\dagger}(q),
\end{aligned}
$$

where $\ell_{\Sigma}+\ell_{p}+J_{B}$ must be even because the spin-flip processes are neglected. This leads to a population of the natural parity states with $J^{\pi}=0^{+}, 1^{-}, 2^{+}, \cdots$. The form factor $F(q)$ is

$$
F(q)=\int_{0}^{\infty} r^{2} d r\left(\tilde{\varphi}_{j_{\Sigma}}(r)\right)^{*} \tilde{j}_{J_{B}}(q, b r) \varphi_{j_{p}}^{(N)}(r)
$$

where $\varphi_{j_{p}}^{(N)}$ is a single-particle wave function for the proton, and $\tilde{\varphi}_{j_{\Sigma}}$ is the biorthogonal one for $\Sigma^{-}$, as given by Eq. (14). The recoil effects are taken into account in the distorted waves of $\tilde{j}_{J_{B}}(q, b r)$ by the factor of $b=M_{C} / M_{B}$ when $M_{B} \simeq M_{A}$ is justified in Eq. (13).

\section{III. $\Sigma$-NUCLEUS POTENTIALS}

We briefly mention the $\Sigma$-nucleus potentials which we discussed in this paper. In previous papers $[7,11]$, we have introduced several types of the $\Sigma$-nucleus potential obtained by fitting to strong-interaction shifts and widths of $\Sigma^{-}$atomic X-ray data; (a) the density-dependent (DD) potential [10], (b) the relativistic mean-field (RMF) potential [38], (c) the local-density approximation potential (LDA-NF) based on YNG-NF interaction [39, 40], (d) the LDA potential (LDA-S3) based on phenomenological two-body $\Sigma N$ SAP-3 interaction [14], (e) the shallow potential in the WS form (WS-sh) [41], and (f) the $t_{\text {eff }} \rho$-type potential $\left(t_{\text {eff }} \rho\right)$ [10]. It should be noticed that all of the potentials sufficiently reproduce the experimental shifts and widths of the $\Sigma^{-}$atomic states; the values of the shifts and widths are mainly sensitive to the tail part of the potentials outside the nuclear surface [10].

\section{FIG. 2}

In Fig. 2, we display the real and imaginary parts of the $\Sigma$-nucleus potentials of DD, LDA-NF, and $t_{\text {eff }} \rho$ for $\Sigma^{-}-^{27} \mathrm{Al}\left(\Sigma_{\Sigma^{-}}^{28} \mathrm{Mg}\right), \Sigma^{-}{ }^{57} \mathrm{Co}\left({ }_{\Sigma^{-}}^{58} \mathrm{Fe}\right)$ and $\Sigma^{-}-^{207} \mathrm{Tl}\left(\Sigma_{\Sigma^{-}}^{208} \mathrm{Hg}\right)$, where the

real parts of the potential include the finite Coulomb potentials. In Refs. [7, 11], it has been attempted to discriminate between these types of the potentials by analyzing the nuclear 
$\left(\pi^{-}, K^{+}\right)$data in the $\Sigma^{-}$continuum spectrum. These analyses have shown that the $\Sigma^{-}$ nucleus potentials have a repulsion inside the nuclear surface and an attraction outside the nucleus, i.e., DD, RMF and LDA-NF, rather than an attraction at the nuclear center, i.e., LDA-S3, WS-sh and $t_{\text {eff }} \rho$. Moreover, the former potentials are considerably different from each other in terms of the repulsion at $r \lesssim R=r_{0}(A-1)^{1 / 3} \mathrm{fm}$ and the attractive pocket outside there. The repulsion in DD at the nuclear center $(\sim 80 \mathrm{MeV})$ is $2-3$ times larger than that in LDA-NF or RMF $(\sim 40 \mathrm{MeV})$ [7], whereas the range of the pockets in DD near the nuclear surface is slightly shorter than that in LDA-NF. Such a difference of the repulsion and attractive pocket is expected to be clearly examined if we can observe the $\Sigma^{-}$bound states in the spectrum. In this paper we will focus on three potentials of DD, LDA-NF and $t_{\text {eff }} \rho$, as the typical examples.

\section{IV. $\quad \Sigma^{-}$-NUCLEUS BOUND STATES}

\section{A. Energies and widths}

In Table II, we show the numerical results of the $\Sigma^{-}$nuclear binding energies and widths of the bound states for $\Sigma^{-}{ }^{27} \mathrm{Al}, \Sigma^{-}-57 \mathrm{Co}$ and $\Sigma^{-}{ }^{207} \mathrm{Tl}$, when we use the DD or $t_{\text {eff }} \rho$ potential including the finite Coulomb potential.

TABLE II

\section{FIG. 3}

For the $t_{\text {eff }} \rho$ potential, we confirm that there exist $\Sigma^{-}$bound states even if the Coulomb potential is switched off (see Fig. 3), because its real part corresponds to $V_{0}^{\Sigma} \simeq-28 \mathrm{MeV}$ as a WS potential, which is similar to the $\Lambda$-nucleus potential $[18,20]$. Such a bound state has a broad width $\left(\Gamma_{\Sigma} / 2 \gtrsim B_{\Sigma^{-}}\right)$, of which the pole arises away from the physical axis in the complex $k$-plane [42]. If the pole is located close to the $\Sigma^{-}$emitted threshold, the magnitude of the production peak is modified by interference effects from background or other pole terms [21]. Thus the shape of the peak in the spectrum does not necessarily correspond to that of a standard Breit-Wigner resonance located at $B_{\Sigma^{-}}[21,24]$.

For the DD potential, on the other hand, we do not obtain the bound states till we take into account the Coulomb attraction because the potential is very repulsive inside the 
nucleus. As seen in Table II, the $\Sigma^{-}$binding energies of $(1 s)_{\Sigma},(1 p)_{\Sigma}$ and $(1 d)_{\Sigma}$ states are significantly shifted upward from those of the finite Coulomb eigenstates (see Fig. 3), and their widths become rather narrow $(\sim 1 \mathrm{MeV})$. A relatively narrow width is obtained in the $\Sigma^{-}$bound states because of the repulsion even if the value of $\left|\operatorname{Im} U_{\Sigma}\right|$ becomes larger. It is recognized that these states are regarded as Coulomb-assisted $\Sigma^{-}$-nucleus bound states. In Fig. 3, we illustrate a summary of the $\Sigma^{-}$nuclear binding energies and widths for low-lying bound states of $\Sigma^{-}{ }^{57}$ Co. The higher excited states look more like $\Sigma^{-}$atomic states and the level density of $\Sigma^{-}$increases toward the $\Sigma^{-}$threshold.

\section{B. Wave functions}

\section{FIG. 4}

To clearly understand the effects of the repulsion such as the DD potential at the nuclear inside, we examine behavior of wave functions of the $\Sigma^{-}$bound states. In Fig. 4, we display the density distributions of $r^{2} \rho_{n \ell}(r)=r^{2}\left|\varphi_{n \ell}(r)\right|^{2}$ that are calculated with the DD or LDA$\mathrm{NF}$ potential, for $(1 s)_{\Sigma},(2 s)_{\Sigma},(1 p)_{\Sigma},(2 p)_{\Sigma},(1 d)_{\Sigma},(2 d)_{\Sigma}$ and $(1 f)_{\Sigma}$ states in $\Sigma^{-}-27 \mathrm{Al}$, $\Sigma^{-}{ }^{57} \mathrm{Co}$ and $\Sigma^{-}{ }^{207} \mathrm{Tl}$. The wave functions of the $(n s)_{\Sigma},(n p)_{\Sigma}$ and $(n d)_{\Sigma}$ states for both DD and LDA-NF are significantly pushed out to the nuclear outside because of the strong repulsion inside the nucleus, in comparison with the Coulomb wave functions. The overlap between wave functions and the imaginary part of the potential is small, so that their widths are considerably reduced. For $\Sigma^{-}{ }^{27} \mathrm{Al}$, indeed, the $(1 s)_{\Sigma}$ state has a narrow width of $\Gamma_{\Sigma}=$ $1.25 \mathrm{MeV}$ and a rms radius of $\left\langle r^{2}\right\rangle_{1 s}^{1 / 2}=9.8 \mathrm{fm}$. For $\Sigma^{-}{ }^{57} \mathrm{Co}$, the $(1 s)_{\Sigma}$ state has $\Gamma_{\Sigma}=1.89$ $\mathrm{MeV}$ and $\left\langle r^{2}\right\rangle_{1 s}^{1 / 2}=9.1 \mathrm{fm}$, and the $(1 p)_{\Sigma}$ state also $\Gamma_{\Sigma}=1.43 \mathrm{MeV}$ and $\left\langle r^{2}\right\rangle_{1 s}^{1 / 2}=9.8 \mathrm{fm}$. If the DD or LDA-NF potential is switched off, we have a rms radius of $\left\langle r^{2}\right\rangle_{1 s}^{1 / 2}=4.2 \mathrm{fm}$ for the $\Sigma^{-}$atomic $(1 s)_{\Sigma}$ state in $\Sigma^{-}{ }^{57} \mathrm{Co}$, of which the core-nuclear radius is $R=r_{0} A^{1 / 3}=$ $4.23 \mathrm{fm}$. The $\Sigma^{-}$hyperon in nuclei is excluded from the nuclear region, and it acts on a strangeness halo in the Coulomb-assisted $\Sigma^{-}$-nucleus bound states.

This nature becomes more conspicuous in heavy nuclei. For $\Sigma^{-}-^{207} \mathrm{Tl}$, wave functions for these states with DD and LDA-NF are extremely pushed out to the nuclear outside because of the strong repulsion inside the nucleus. The binding energy and a rms radius of the $(1 s)_{\Sigma}$ state for DD are $B_{\Sigma^{-}}=-10.5 \mathrm{MeV}$ and $\left\langle r^{2}\right\rangle_{1 s}^{1 / 2}=9.8 \mathrm{fm}$, respectively, in contrast to $B_{\Sigma^{-}}=$ $-18.8 \mathrm{MeV}$ and $\left\langle r^{2}\right\rangle_{1 s}^{1 / 2}=4.2 \mathrm{fm}$ in only the Coulomb $(1 s)_{\Sigma}$ state. If the $\Sigma^{-}$narrow bound 
state with a width of $\Gamma_{\Sigma}=1.7 \mathrm{MeV}$ can be selectively populated, one expects to clearly see a distinct peak of the state in the $\Sigma^{-}$bound region. For details of the repulsive component in the $\Sigma$-nucleus potential, moreover, it is important to find a quantitative different signal between DD and LDA-NF in the calculated spectrum of the $\Sigma^{-}$bound states. As shown in Fig. 4, however, a discrepancy between these wave functions for $\Sigma^{-}{ }^{207} \mathrm{Tl}$ is rather small because these wave functions are predominately located near the attractive pocket formed by the Coulomb potential. Consequently, it might be difficult to distinguish the repulsion inside the nuclei between the DD and LDA-NF potentials for heavy nuclei such as $\Sigma^{-} \_{ }^{207} \mathrm{Tl}$ systems. The production spectrum for the $\left(K^{-}, \pi^{+}\right)$reaction on a ${ }^{208} \mathrm{~Pb}$ target will be discussed in Sect. V C.

\section{RESULTS AND DISCUSSION}

As mentioned in Sect. I, the $\left(K^{-}, \pi^{+}\right)$reaction provides the ability of a production of "substitutional states" under the recoilless condition, in contrast to the exothermic $\left(\pi^{-}, K^{+}\right)$ reactions. Because the $K^{-}+p \rightarrow \pi^{+}+\Sigma^{-}$spin-flip process is negligible in this momentum region, the $\left(K^{-}, \pi^{+}\right)$reaction on the closed-shell nuclear targets with $J^{\pi}=0^{+}$can selectively populate natural-parity states with $J^{\pi}=0^{+}, 1^{-}, 2^{+}, \cdots$, as seen in Eq. (22), in only the isospin transfer $\Delta T=3 / 2$. Thus this reaction is advantageous to populate the Coulomb-assisted $\Sigma^{-}$bound states in the nucleus [43]. We expect to extract more quantitative information on a repulsive component of the $\Sigma$-nucleus potential from the nuclear $\left(K^{-}, \pi^{+}\right)$spectrum, by choosing an appropriate target nucleus and kinematics. Now we examine the production spectra of the Coulomb-assisted $\Sigma^{-}$-nucleus bound states by the $\left(K^{-}, \pi^{+}\right)$reactions on ${ }^{28} \mathrm{Si},{ }^{58} \mathrm{Ni}$, and ${ }^{208} \mathrm{~Pb}$ targets. In this paper, we assume a detector resolution of $1.5 \mathrm{MeV}$ FWHM for the following calculated spectra.

\section{A. ${ }^{58} \mathrm{Ni}\left(K^{-}, \pi^{+}\right)$spectrum}

Let us consider the Coulomb-assisted $\Sigma^{-}$-nucleus bound states by the $\left(K^{-}, \pi^{+}\right)$reaction on the ${ }^{58} \mathrm{Ni}$ target. The nucleus ${ }^{58} \mathrm{Ni}$ is very suitable because it is nearly a subclosed-shell nucleus of the proton $f_{7 / 2}$ orbit, and its proton-hole strength is well concentrated on the $J^{\pi}=7 / 2^{-}, T=3 / 2$ ground state of ${ }^{57} \mathrm{Co}[44]$. Thus the ${ }_{\Sigma^{-}}^{58}$ Fe hypernucleus, which consists 
of the $\Sigma^{-}$and the ${ }^{57}$ Co nucleus, can be produced with total isospin $T=5 / 2, T_{z}=-5 / 2$. As seen in Fig. 3, the energies and widths of the $\Sigma^{-}-58$ Co bound states with the DD potential are very different from those with the $t_{\text {eff }} \rho$ potential, as well as those with only the finite Coulomb one. To establish properties of the $\Sigma$-nucleus potentials for $\Sigma^{-}{ }^{57} \mathrm{Co}$, we demonstrate the production spectra of the $\Sigma^{-}$-nucleus bound states by utilizing the near-recoilless $\left(K^{-}, \pi^{+}\right)$ reaction.

\section{Strength function}

By the Green's function technique, we calculate the inclusive spectra of the $\Sigma^{-}-57$ Co bound states with the DD potential in the ${ }^{58} \mathrm{Ni}\left(K^{-}, \pi^{+}\right)$reaction. To clarify the dependence of the spectrum on the momentum transfer $q_{\Sigma}$, we evaluate the strength function of $S(\omega)$ in Eq. (12). In Fig. 5, we show the strength functions $S(\omega)$ near the $\Sigma^{-}$threshold on the incident $K^{-}$lab momenta of (a) $p_{K}=260 \mathrm{MeV} / \mathrm{c}$, (b) $400 \mathrm{MeV} / \mathrm{c}$, (c) $600 \mathrm{MeV} / \mathrm{c}$ and (d) $800 \mathrm{MeV} / \mathrm{c}$ with the angle $\theta_{\text {lab }}=5^{\circ}$, which correspond to the momentum transfers of $q_{\Sigma}=22 \mathrm{MeV} / \mathrm{c}, 66 \mathrm{MeV} / \mathrm{c}, 117 \mathrm{MeV} / \mathrm{c}$ and $153 \mathrm{MeV} / \mathrm{c}$, respectively (see Fig. 1(b)). The kinematical factor $\beta$ is taken to be $\beta=1.02-0.89$ depending on $-B_{\Sigma^{-}}=(-30)-(+20) \mathrm{MeV}$ at $600 \mathrm{MeV} / \mathrm{c}\left(5^{\circ}\right)$. The inclusive cross sections can be obtained by Eq. (11).

\section{FIG. 5}

For $p_{K}=260 \mathrm{MeV} / \mathrm{c}\left(q_{\Sigma}=22 \mathrm{MeV} / \mathrm{c}\right)$, which leads to an almost recoilless kinematics, we find that a series of $f_{\Sigma}$ states involving the higher-excited $\Sigma^{-}$atomic bound and continuum states with a couple to an $f_{7 / 2}^{-1}$ proton-hole state is strongly favored because a $\Delta L=0$ transition is dominant, as well as a substitutional $\left(1 f_{7 / 2}^{-1}, 1 f_{\Sigma}\right)_{0^{+}}$state at $B_{\Sigma^{-}}=1.43 \mathrm{MeV}$. Such a spectrum is rather unsuitable to see the $\Sigma^{-}$nuclear bound states to extract the repulsive component of the $\Sigma$-nucleus potential. The value of $\langle d \sigma / d \Omega\rangle_{\text {lab }}^{K^{-} p \rightarrow \pi^{+} \Sigma^{-}}$is experimentally unknown (see Fig. 1(a)), and a corresponding measurement seems to be difficult due to a background of $K^{-} \rightarrow \pi^{+} \pi^{-} \pi^{-}$decays in the low momentum $K^{-}$beams at the forward direction. For $p_{K}=400 \mathrm{MeV} / \mathrm{c}\left(q_{\Sigma} \simeq 66 \mathrm{MeV} / \mathrm{c}\right)$ where $\langle d \sigma / d \Omega\rangle_{\mathrm{lab}}^{K^{-} p \rightarrow \pi^{+} \Sigma^{-}}=1.13 \mathrm{mb} / \mathrm{sr}$, we find that the partial-wave components of $\left(s_{1 / 2}\right)_{\Sigma},\left(p_{3 / 2}\right)_{\Sigma},\left(d_{5 / 2}\right)_{\Sigma}$ and $\left(f_{7 / 2}\right)_{\Sigma}$ are fairly populated with a couple to an $f_{7 / 2}^{-1}$ proton-hole state, leading to a bump with $-B_{\Sigma^{-}} \simeq-3.5$ $\mathrm{MeV}$ and $\Gamma_{\Sigma} \simeq 4 \mathrm{MeV}$. Although $q_{\Sigma} \simeq 66 \mathrm{MeV} / \mathrm{c}$ is still small, the peak for the $\left(f_{7 / 2}\right)_{\Sigma}$ state 
is reduced and the bump structure is formed in the spectrum. For $p_{K}=600 \mathrm{MeV} / \mathrm{c}\left(q_{\Sigma} \simeq\right.$ $117 \mathrm{MeV} / \mathrm{c}$ ) where $\langle d \sigma / d \Omega\rangle_{\mathrm{lab}}^{K^{-} p \rightarrow \pi^{+} \Sigma^{-}}=1.25 \mathrm{mb} / \mathrm{sr}$ is near its maximum, we show that the bump structure is mainly constructed with partial-wave components of $\left(s_{1 / 2}\right)_{\Sigma},\left(p_{3 / 2}\right)_{\Sigma}$ and $\left(d_{5 / 2}\right)_{\Sigma}$, but the contribution of the $\left(f_{7 / 2}\right)_{\Sigma}$ component is small. Thus the bump structure appears more clearly below the $\Sigma^{-}$threshold in the spectrum. For $p_{K}=800 \mathrm{MeV} / \mathrm{c}\left(q_{\Sigma} \simeq 153\right.$ $\mathrm{MeV} / \mathrm{c})$, we find that the contributions of $\left(d_{5 / 2}\right)_{\Sigma}$ and $\left(d_{3 / 2}\right)_{\Sigma}$ to the bump are comparable, and the dip around $-B_{\Sigma^{-}} \simeq-2 \mathrm{MeV}$ tends to be filled by other partial-wave contributions. Consequently, the momentum transfer of the near-recoilless $q_{\Sigma} \simeq 110 \mathrm{MeV} / \mathrm{c}$ at $p_{K} \simeq 600$ $\mathrm{MeV} / \mathrm{c}$ is necessary to clearly obtain the bump structure of the Coulomb-assisted $\Sigma^{-}$bound states in the spectrum.

\section{FIG. 6}

To see the angular dependence of the spectrum, we calculate the inclusive spectra at $p_{K}=600 \mathrm{MeV} / \mathrm{c}$, as a function of $\theta_{\text {lab. }}$. In Fig. 6 , we show the spectra at $\theta_{\text {lab }}=5^{\circ}, 10^{\circ}, 20^{\circ}$ and $30^{\circ}$, which correspond to $q_{\Sigma} \simeq 117,143,214$ and $296 \mathrm{MeV} / \mathrm{c}$, respectively. A structure of the peak and dip below the $\Sigma^{-}$threshold in the spectrum disappears as increasing $q_{\Sigma}$. The shape of the spectrum at $\theta_{\text {lab }}=30^{\circ}$ in which $q_{\Sigma} \simeq 296 \mathrm{MeV} / \mathrm{c}$ is near by the Fermi momentum of $\sim 270 \mathrm{MeV} / \mathrm{c}$, becomes similar to that of the $\left(\pi^{-}, K^{+}\right)$reaction with a high momentum transfer of $\sim 380 \mathrm{MeV} / \mathrm{c}$. Therefore, the spectrum at $p_{K} \simeq 600 \mathrm{MeV} / \mathrm{c}\left(5^{\circ}\right)$ is expected to be a complement to the analyses of the $\Sigma^{-}$atomic and $\left(\pi^{-}, K^{+}\right)$data.

\section{Comparison between the inclusive spectra for various $\Sigma$-nucleus potentials}

\section{FIG. 7}

In Fig. 7, we show a comparison of the calculated spectra of the ${ }^{58} \mathrm{Ni}\left(K^{-}, \pi^{+}\right)$reaction at $p_{K}=600 \mathrm{MeV} / \mathrm{c}\left(5^{\circ}\right)$, using all the types of the potentials mentioned in Sect. III. For comparison with the shapes of the spectra each other, we normalize these spectra to the value calculated by DD at $\omega=277.3 \mathrm{MeV}$ (see values of the normalization factors $f_{s}$ in Fig. 7). It is clearly shown that the spectra with the potentials having a repulsion inside the nucleus such as DD, RMF and LDA-NF, are significantly different from those with the

potentials having some attractions inside the nucleus such as LDA-S3, WS-sh and $t_{\text {eff }} \rho$. The $\left(K^{-}, \pi^{+}\right)$spectra with a low momentum transfer $q_{\Sigma} \simeq 110 \mathrm{MeV} /$ c provides clear evidence 
to qualitatively identify the different type of the $\Sigma$-nucleus potentials. This fact implies that the near-recoilless $\left(K^{-}, \pi^{+}\right)$spectra confirm the repulsive components of the potential inside the nucleus, as a complement to $\Sigma^{-}$atomic and $\left(\pi^{-}, K^{+}\right)$data. Such a experiment is expected to observe a promising spectrum of the nuclear $\left(K^{-}, \pi^{+}\right)$reaction at J-PARC facilities.

However, we must recognize that the shape and magnitude of the spectra with the potentials for DD, RMF and LDA-NF are quite similar to each other, regardless of a discrepancy between the radial distributions of these potentials. The main reason originates from the appearance of the sizable absorption of $\operatorname{Im} U_{\Sigma}=(-20)-(-30) \mathrm{MeV}$. It would be difficult to determine the strength of the repulsion at the nuclear center from the inclusive $\left(K^{-}, \pi^{+}\right)$ spectrum.

\section{Integrated cross sections}

To study the structure of the Coulomb-assisted $\Sigma^{-}$-nucleus bound states, we also calculate the integrated cross sections, which can be intuitively understood by the behavior of $\Sigma^{-}$wave functions, as seen in Eqs. (22) and (23).

FIG. 8

TABLE III

Firstly, we start to consider the $\Sigma^{-}$bound states with the $t_{\text {eff }} \rho$ potential, because this situation is analogous to the $\Lambda$-hypernuclear production in the $\left(K^{-}, \pi^{-}\right)$reaction, except their widths. In Fig. 8, we illustrate the results of the integrated cross sections of the $\Sigma^{-}$ bound states with $\left(j_{p}^{-1}, n \ell_{\Sigma}\right)_{J^{\pi}}$ for $\Sigma^{-}{ }^{57} \mathrm{Co}$ in the ${ }^{58} \mathrm{Ni}\left(K^{-}, \pi^{+}\right)$reactions at $p_{K}=400,600$ and $800 \mathrm{MeV} / \mathrm{c}\left(5^{\circ}\right)$. Here we estimated them with only the real part of the potential to clearly see their dependence on $q_{\Sigma}$ when we took into account the $1 f_{7 / 2}^{-1}$ and $2 s_{1 / 2}^{-1}$ proton-hole states.

As far as a recoilless kinematics, we expect that "substitutional states" are selectively populated in the $\Sigma^{-}$bound states, e.g., $\left(1 f_{7 / 2}^{-1}, 1 f_{\Sigma}\right)_{0^{+}}$with the $\Delta L=0$ transition. This nature approximates to a production at $400 \mathrm{MeV} / \mathrm{c}$ with $q_{\Sigma} \simeq 66 \mathrm{MeV} / \mathrm{c}$, as seen in Fig. 8 . The components of the $\left(1 f_{7 / 2}^{-1}, 1 d_{\Sigma}\right)_{3^{-}, 5^{-}}$states are enhanced as increasing $q_{\Sigma}$, whereas the 
components of $\left(1 f_{7 / 2}^{-1}, 1 f_{\Sigma}\right)_{0^{+}}$are reduced. We confirm that the partial-wave components in the cross section are very sensitive to the value of the momentum transfer. This behavior is reasonable from the viewpoint of the $\Lambda$-hypernuclear production [20]. However, this scenario is changed for the DD potential, as we will discuss below. In Table III, we list the values of these cross sections with $q_{\Sigma} \simeq 117 \mathrm{MeV} / \mathrm{c}$ at $p_{K}=600 \mathrm{MeV} / \mathrm{c}\left(5^{\circ}\right)$, together with the complex effective numbers of a proton, $P_{\text {eff }}$. To see the effects of the attraction in the real part of the potential, we also show the calculated results when we omit the imaginary part of the potential (real only). We evaluate their $\operatorname{argument,} \operatorname{Re} P_{\text {eff }}$ and $\operatorname{Arg} P_{\text {eff }}$, in order to understand the shape of the inclusive spectrum where the imaginary part of the potential is switched on (full).

FIG. 9

\section{TABLE IV}

Secondly, we consider the $\Sigma^{-}$bound states with the DD potential. In Fig. 9, we illustrate the results of integrated cross sections for the $\Sigma^{-}$bound states in ${ }^{58} \mathrm{Ni}\left(K^{-}, \pi^{+}\right)$reactions at $p_{K}=400,600$ and $800 \mathrm{MeV} / \mathrm{c}\left(5^{\circ}\right)$. We find that the contributions of $\left(1 f_{7 / 2}^{-1}, 1 p_{\Sigma}\right)_{2^{+}, 4^{+}}$ states with $\Delta L=2$ are populated dominantly, and also $\left(1 f_{7 / 2}^{-1}, 1 d_{\Sigma}\right)_{1^{-}, 3^{-}}$states with $\Delta L=1$ even if $p_{K}=400 \mathrm{MeV} / \mathrm{c}$. This originates predominately from a large difference between the wave functions of the $(1 d)_{\Sigma}$ or $(1 f)_{\Sigma}$ state and the proton-hole $1 f_{7 / 2}^{-1}$ state, as seen in Fig. 4 . The high momentum components caused by the repulsion require a $\Delta L \neq 0$ transition to populate the $\Sigma^{-}$bound states. The distorted waves of $\tilde{j}_{L}(q, b r)$ in Eq. (5) also moderate the pattern of the population. In Table IV, we show that $\operatorname{Re} P_{\text {eff }}$ is reduced with $\operatorname{Arg} P_{\text {eff }}=$ $45-55^{\circ}$ in the complex effective number approach [24]. To see the effects of the repulsion in the real part of the potential, we also list the calculated results without the imaginary part of the potential. Such a peak is shifted downward from the energy position at $-B_{\Sigma^{-}}$and behaves as an asymmetric shape with a relatively narrow width [35].

\section{B. ${ }^{28} \operatorname{Si}\left(K^{-}, \pi^{+}\right)$spectrum}

FIG. 10

We attempt to examine a production of the $\Sigma^{-}$bound states for $\Sigma^{-}{ }^{27} \mathrm{Al}$ in the ${ }^{28} \mathrm{Si}\left(K^{-}, \pi^{+}\right)$reaction with a small momentum transfer of $q_{\Sigma} \simeq 110 \mathrm{MeV} / \mathrm{c}$. In Fig. 10, 
we show a comparison of the calculated inclusive $\left(K^{-}, \pi^{+}\right)$spectra near the $\Sigma^{-}$threshold at $600 \mathrm{MeV} / \mathrm{c}\left(5^{\circ}\right)$ as a function of the energy transfer $\omega$. Here we used several types of the potentials that were tested by the $\left(\pi^{-}, K^{+}\right)$reactions in Ref. [7], as mentioned in Sect. III. Note that these spectra were renormalized to the value obtained by the DD potential at $\omega=$ $280.8 \mathrm{MeV}$.

We find that a peak of $\left(1 d_{5 / 2}^{-1}, 1 p_{\Sigma}\right)_{1^{-}, 3^{-}}$is fairly enhanced at $\omega \simeq 269 \mathrm{MeV}$ below the $\Sigma^{-}$ threshold in the spectra for the almost types of the potential. The calculated spectra with the repulsive potentials (DD, RMF and LDA-NF) significantly differ from those with the attractive ones (LDA-S3, WS-sh and $t_{\text {eff }} \rho$ ). Therefore, the discrepancy becomes much more clear by comparing the shape behavior of each spectrum in the bound region; the absolute values of the former cross sections are scarcely smaller than those of the latter ones, as seen by the normalization factors $f_{s}$ in Fig. 10 .

The $\Sigma^{-}$energy levels are pushed up by this repulsion, so that the level spacing becomes tight. Since the binding energy of the $(1 p)_{\Sigma}$ bound state is $B_{\Sigma^{-}}=1.13 \mathrm{MeV}$ for DD in Tables II, which is very different from $B_{\Sigma^{-}}=9.54 \mathrm{MeV}$ for $t_{\mathrm{eff}} \rho$, the gap energy of the levels between the other bound states is not so large. Consequently, the attraction for $\Sigma^{-}$ with the help of the Coulomb potential is more needed to clearly identify a $\Sigma^{-}$bound state. We believe that it is not so difficult to measure a peak structure of the Coulomb-assisted $\Sigma^{-}$-nucleus bound states in heavier systems such as $\Sigma^{-}{ }^{57} \mathrm{Co}$, rather than $\Sigma^{-}{ }^{27} \mathrm{Al}$.

\section{C. ${ }^{208} \mathrm{~Pb}\left(K^{-}, \pi^{+}\right)$spectrum}

To see whether we tell the difference of the repulsive nature between DD and LDA-NF or not, we consider the spectrum of the Coulomb-assisted $\Sigma^{-}$bound states in the heavy nuclei. In Fig. 2, we represent the real and imaginary parts of the $\Sigma^{-}{ }^{207} \mathrm{Tl}$ potentials for DD and LDA-NF, together with the finite Coulomb potential. The Coulomb potential gives a large contribution to the potential energy at the nuclear center, i.e., $\left|U_{\text {Coul }}\right| \simeq 25 \mathrm{MeV}$, which is comparable to that from the repulsion in LDA-NF. As seen in Fig. 4, wave functions for the $(n s)_{\Sigma},(n p)_{\Sigma}$ and $(n d)_{\Sigma}$ states are strongly modified by the appearance of the repulsion in comparison with the Coulomb wave functions. The repulsion in DD at the nuclear center $\left(\operatorname{Re} U_{\Sigma} \sim 80 \mathrm{MeV}\right)$ is 2-3 times larger than that in RMF or LDA-NF $\left(\operatorname{Re} U_{\Sigma} \sim 40 \mathrm{MeV}\right)$. 
Let us consider the inclusive ${ }^{208} \mathrm{~Pb}\left(K^{-}, \pi^{+}\right)$spectra at $600 \mathrm{MeV} / \mathrm{c}\left(5^{\circ}\right)$. Here we use the DD, LDA-NF and $t_{\text {eff }} \rho$ potentials. These types of the potential were also used in calculations of ${ }^{209} \mathrm{Bi}\left(\pi^{-}, K^{+}\right)$reactions [11]. In Fig. 11, we show a comparison between the calculated inclusive spectra of ${ }^{208} \mathrm{~Pb}\left(K^{-}, \pi^{+}\right)$reactions, taking into account 16 proton-hole states involving $1 h_{11 / 2}^{-1}, 1 g_{9 / 2}^{-1}$ and $2 d_{5 / 2}^{-1}$ proton-hole ones. We find that the shape of the calculated spectra clearly discriminate between the repulsive potentials (DD, LDA-NF) and the attractive one $\left(t_{\mathrm{eff}} \rho\right)$, but the spectra for DD and LDA-NF are very similar to each other. The insensitivity of the spectrum to the real part of the potential is caused by the following reason. The ratio of the expectation values of the $(1 s)_{\Sigma}$ state for $\Sigma^{-}{ }^{207} \mathrm{Tl}$,

$$
\left|\frac{\left\langle\operatorname{Re} U_{\Sigma}\right\rangle}{\left\langle\operatorname{Re} U_{\Sigma}+U_{\text {Coul }}\right\rangle}\right|=0.025 \quad(0.009)
$$

in DD (LDA-NF) is suppressed rather than 0.131 (0.088) for $\Sigma^{-}-^{27} \mathrm{Al}$. Unfortunately, we recognize that the effect of the $\Sigma$-nucleus potential is rather masked by the Coulomb potential due to the heaver nuclei.

As shown in Fig. 11, many partial-wave states of the Coulomb-assisted $\Sigma^{-}$-nucleus states can be easily populated by the $\left(K^{-}, \pi^{+}\right)$reaction on ${ }^{208} \mathrm{~Pb}$. In contribution from a $1 h_{11 / 2}^{-1}$ proton-hole state, the first peak at $\omega \simeq 258 \mathrm{MeV}$ is constructed with $(1 s)_{\Sigma},(1 p)_{\Sigma}$ and $(1 d)_{\Sigma}$ states, whereas the second peak at $\omega \simeq 263 \mathrm{MeV}$ with $(1 h)_{\Sigma},(2 p)_{\Sigma},(2 d)_{\Sigma}$ and $(2 f)_{\Sigma}$ states. In ${ }^{208} \mathrm{~Pb}$, individual levels with broad widths are covering each other in $\left(j_{p}^{-1}, n \ell_{\Sigma}\right)$ configurations because the level spacing of the populated $\Sigma^{-}$states is very tight for the Coulomb attraction. Moreover, the shape and magnitude of these spectra are masked by contributions of other proton-hole states [45] that can couple with a lot of $\Sigma^{-}$states, e.g., $3 s_{1 / 2}^{-1}, 2 d_{5 / 2,3 / 2}^{-1}$ and $1 g_{9 / 2,7 / 2}^{-1}$. This results are contrast to early calculations [43] in which only the $1 h_{11 / 2}^{-1}$ proton-hole state was considered. It should be noticed that the contributions of the deep-hole states are important to examine the spectrum below the $\Sigma^{-}$threshold quantitatively. This implies the importance of choosing the appropriate nucleus as a target, e.g., ${ }^{58} \mathrm{Ni}$, as discussed in Sect. V A.

\section{SUMMARY AND CONCLUSION}

We have performed the theoretical study of the Coulomb-assisted $\Sigma^{-}$-nucleus bound states to examine properties of the $\Sigma$-nucleus potential and have attempted to discriminate 
between various types of the $\Sigma$-nucleus potentials that are consistent with the $\Sigma^{-}$atomic X-ray and nuclear $\left(\pi^{-}, K^{+}\right)$data. Some potentials we used are repulsive inside the nuclear surface and attractive outside the nucleus with the sizable absorption. We have calculated the DWIA inclusive spectra in the $\left(K^{-}, \pi^{+}\right)$reactions on ${ }^{28} \mathrm{Si},{ }^{58} \mathrm{Ni}$ and ${ }^{208} \mathrm{~Pb}$ targets at $p_{K} \simeq 600 \mathrm{MeV} / \mathrm{c}$ by using several types of the $\Sigma$-nucleus potential. We have predicted the shape and magnitude of the inclusive spectra for the Coulomb-assisted $\Sigma^{-}$-nucleus bound states under appropriate kinematics for the forthcoming J-PARC experiments. The results are summarized as follows:

(i) The nuclear $\left(K^{-}, \pi^{+}\right)$reaction with the momentum transfer of $q_{\Sigma} \simeq 110 \mathrm{MeV} / \mathrm{c}$ discriminates more clearly between properties of the $\Sigma$-nucleus potentials, e.g., repulsive or attractive.

(ii) The bump structure formed by $\left(s_{1 / 2}\right)_{\Sigma},\left(p_{3 / 2}\right)_{\Sigma}$ and $\left(d_{5 / 2}\right)_{\Sigma}$ components of $\Sigma^{-}{ }^{57} \mathrm{Co}$ is expected to be observed in the inclusive $\left(K^{-}, \pi^{+}\right)$spectrum on ${ }^{58} \mathrm{Ni}$ at $600 \mathrm{MeV} / \mathrm{c}$ $\left(5^{\circ}\right)$, which is shown by the calculation with the repulsive $\Sigma$-nucleus potential such as DD.

(iii) Details of the radial distributions in the $\Sigma$-nucleus potential inside the nucleus and its repulsive component at the center are still not determined because of the sizable absorption.

(iv) For the heavier nuclei, the shape and magnitude of the spectrum near the $\Sigma^{-}$threshold are rather insensitive to the $\Sigma$-nucleus potential because the level spacing of the populated $\Sigma^{-}$states is very tight for the Coulomb attraction.

In conclusion, the near-recoilless $\left(K^{-}, \pi^{+}\right)$reactions on suitable nuclear targets such as ${ }^{58} \mathrm{Ni}$ provide a candidate to clearly discriminate between qualitative properties of the $\Sigma$ nucleus potentials, which can populate the Coulomb-assisted $\Sigma^{-}$-nucleus bound states with a relatively narrow peak at the forthcoming J-PARC experiments. We believe that properties of the $\Sigma$-nucleus potential are quantitatively clarified by obtaining valuable information on the production of the Coulomb-assisted $\Sigma^{-}$-nucleus bound states in the $\left(K^{-}, \pi^{+}\right)$reactions, as a full complement to the energy-shifts and widths of $\Sigma^{-}$atomic X-ray data and the $\Sigma^{-}$ QF spectrum of $\left(\pi^{-}, K^{+}\right)$data. 


\section{Acknowledgments}

The authors are obliged to Professor A. Gal and Dr. J. Mareš for valuable comments,

and are also grateful to Dr. T. Koike, Dr. A. Umeya, Professor Y. Akaishi and Professor T. Fukuda for useful discussion. This work is supported by Grant-in-Aid for Scientific Research on Priority Areas (No. 17070007).

[1] S. Balberg and A. Gal, Nucl. Phys. A625 (1997) 435.

[2] A. Gal and C. B. Dover, Phys. Rev. Lett. 44 (1980) 379.

[3] E. Friedman and A. Gal, Phys. Rept. 452 (2007) 89, and references therein.

[4] H. Noumi, P. K. Saha, D. Abe, S. Ajimura, K. Aoki, H. C. Bhang, T. Endo, Y. Fujii, T. Fukuda, H. C. Guo, K. Imai, O. Hashimoto, H. Hotchi, E. H. Kim, J. H. Kim, T. Kishimoto, A. Krutenkova, K. Maeda, T. Nagae, M. Nakamura, H. Outa, M. Sekimoto, T. Saito, A. Sakaguchi, Y. Sato, R. Sawafta, and Y. Shimizu, Phys. Rev. Lett. 89 (2002) 072301; 90 (2003) 049902, Erratum.

[5] P. K. Saha, H. Noumi, D. Abe, S. Ajimura, K. Aoki, H. C. Bhang, K. Dobashi, T. Endo, Y. Fujii, T. Fukuda, H. C. Guo, O. Hashimoto, H. Hotchi, K. Imai, E. H. Kim, J. H. Kim, T. Kishimoto, A. Krutenkova, K. Maeda, T. Nagae, M. Nakamura, H. Outa, T. Saito, A. Sakaguchi, Y. Sato, R. Sawafta, and M. Sekimoto, Phys. Rev. C70 (2004) 044613.

[6] M. Kohno, Y Fujiwara, Y. Watanabe, K. Ogata, and M. Kawai, Prog. Theor. Phys. 112 (2004) 895.

[7] T. Harada and Y. Hirabayashi, Nucl. Phys. A759 (2005) 143.

[8] Y. Fujiwara, Y. Suzuki, and C. Nakamoto, Prog. Part. Nucl. Phys. 58 (2007) 439.

[9] T. A. Rijken and Y. Yamamoto, Nucl. Phys. A804 (2008) 51.

[10] C. J. Batty, E. Friedman, and A. Gal, Prog. Theor. Phys. Suppl. 117 (1994) 227; Phys. Lett. B335 (1994) 273.

[11] T. Harada and Y. Hirabayashi, Nucl. Phys. A767 (2006) 206 .

[12] T. Yamazaki, R. S. Hayano, O. Morimatsu, and K. Yazaki, Phys. Lett. B207 (1988) 393.

[13] R. H. Dalitz and A. Gal, Ann. Phys. (N.Y.) 116 (1978) 167.

[14] T. Harada, Proceedings of the 23rd INS International Symposium on Nuclear and Particle 
Physics with Meson Beams in the 1 GeV/c Region, Tokyo, March, 1995 (Universal Academy Press, Inc., Tokyo, 1995) p. 211 (1995).

[15] J. Hüfner, S. Y. Lee, and H. A. Weidenmüller, Nucl. Phys. A234 (1974) 429.

[16] A. Bouyssy, Nucl. Phys. A290 (1977) 324.

[17] E. H. Auerbach, A. J. Baltz, C. B. Dover, A. Gal, S. H. Kahana, L. Ludeking, and D. J. Millener, Ann. Phys. (N.Y.) 148 (1983) 381.

[18] C. B. Dover, L. Ludeking, and G. E. Walker, Phys. Rev. C22 (1980) 2073.

[19] C. B. Dover and A. Gal, Ann. Phys. (N.Y.) 146 (1983) 309.

[20] T. Motoba, H. Bandō, R. Wünsch, and J. Žofka, Phys. Rev. C38 (1988) 1322.

[21] O. Morimatsu and K. Yazaki, Nucl. Phys. A483 (1988) 493.

[22] C. B. Dover, D. J. Millener, and A. Gal, Phys. Rep. 184 (1989) 1.

[23] S. Tadokoro, H. Kobayashi, Y. Akaishi, Phys. Rev. C51 (1995) 2656.

[24] T. Koike and T. Harada, Nucl. Phys. A804 (2008) 231.

[25] T. Harada and Y. Hirabayashi, Nucl. Phys. A744 (2004) 323.

[26] H. de Vries, C. W. de Jager, and C. de Vries, At. Data Nucl. Tables 36 (1987) 459.

[27] A. Bohr and M. Mottelson, Nuclear structure, Vol. 1, p.238. (Benjemin, New York, 1969).

[28] G. Jacob and T.A.J. Maris, Rev. Mod. Phys. 38 (1966) 121.

[29] A.E.L. Dieperink and P.K.A. DeWitt Huberts, Annu. Rev. Nucl. Part. Sci. 40 (1990) 239.

[30] D. Vautherin and D.M. Brink, Phys. Rev. C5 (1972) 626.

[31] B. W. Allardyce, C. J. Batty, D. J. Baugh, E. Friedman, G. Heymann, M. E. Cage, Pyle G. J., G. T. A. Squier, A. S. Clough, D. F. Jackson, S. Murugesu, and V. Rajaratnam, Nucl. Phys. A209 (1973) 1.

[32] A. S. Rosenthal and F. Tabakin, Phys. Rev. C22 (1980) 711.

[33] G. P. Gopal, R. T. Ross, A. J. van Horn, A. C. McPherson, E. F. Clayton, T. C. Bacon, and I. Butterworth, Nucl. Phys. B119 (1977) 362.

[34] C. B. Dover, A. Gal, G. E. Walker, and R. H. Dalitz, Phys. Lett. 89 (1979) 26.

[35] O. Morimatsu and K. Yazaki, Prog. Part. Nucl. Phys. 33 (1994) 679.

[36] T. Berggren, Nucl. Phys. A109 (1968) 265.

[37] P.L. Kapur and R. Peierls, Proc. R. Soc. London A166 (1938) 277.

[38] J. Mareš, E. Friedman, A. Gal, and B. K. Jennings, Nucl. Phys. A594 (1995) 311.

[39] J. Dąbrowski, J. Rożynek, and G.S. Anagnostatos, Eur. Phys. J. A14 (2002) 125. 
[40] Y. Yamamoto and H. Bandō, Prog. Theor. Phys. Suppl. 81 (1985) 9; Y. Yamamoto, T. Motoba, H. Himeno, K. Ikeda, and S. Nagata, Prog. Theor. Phys. Suppl. 117 (1994) 241.

[41] R. S. Hayano, Nucl. Phys. A478 (1988) 113c.

[42] A. Gal, G. Toker, and Y. Alexander, Ann. Phys. 137 (1981) 341.

[43] S. Tadokoro and Y. Akaishi, Phys. Rev. C42 (1990) 2591; H. Kobayashi, Master's thesis, University of Tokyo, 1995.

[44] A. Marinov, W. Oelert, S. Gopal, B. Brinkmöller, G. Hlawatsch, C. Mayer-Böricke, J. Meissburger, D. Paul, M. Rogge, J. G. M. Römer, J. L. Tain, P. Turek, L. Zemlo, R. B. M. Mooy, P. W. M. Glaudemans, S. Brant, V. Paar, M. Vouk, V. Lopac, Nucl. Phys. A438 (1985) 429.

[45] W. C. Parkinson, D. L. Hendrie, H. H. Duhm, J. Mahoney, J. Saundinos, and G. R. Satchler, Phys. Rev. 178 (1969) 1976. 
TABLE I: Energies of single-particle states for a proton in ${ }^{28} \mathrm{Si},{ }^{58} \mathrm{Ni}$ and ${ }^{208} \mathrm{~Pb}$ targets, which are input in this calculation [28-30]. The values in parentheses denote width of a deep-hole state for the proton. All energies and widths are in $\mathrm{MeV}$.

\begin{tabular}{|c|c|c|c|c|c|c|}
\hline \multirow{2}{*}{$\frac{(n \ell j)_{p}^{-1}}{1 s_{1 / 2}}$} & \multicolumn{2}{|c|}{${ }^{28} \mathrm{Si}$} & \multicolumn{2}{|c|}{${ }^{58} \mathrm{Ni}$} & \multicolumn{2}{|c|}{${ }^{208} \mathrm{~Pb}$} \\
\hline & -41 & $(10.0)$ & -41.9 & $(10.0)$ & -36.6 & (10.0) \\
\hline $1 p_{3 / 2}$ & -23 & $(6.0)$ & -30.8 & $(10.0)$ & -33.1 & $(10.0)$ \\
\hline $1 p_{1 / 2}$ & -16 & $(4.0)$ & -28.9 & $(8.0)$ & -32.5 & $(10.0)$ \\
\hline $1 d_{5 / 2}$ & -11.6 & $(0.0)$ & -19.2 & $(4.0)$ & -28.4 & $(6.0)$ \\
\hline $1 d_{3 / 2}$ & & & -14.5 & $(2.0)$ & -27.0 & $(6.0)$ \\
\hline $2 s_{1 / 2}$ & & & -14.6 & $(2.0)$ & -24.0 & $(6.0)$ \\
\hline $1 f_{7 / 2}$ & & & -7.8 & $(0.0)$ & -22.9 & $(6.0)$ \\
\hline $1 f_{5 / 2}$ & & & & & -20.4 & $(4.0)$ \\
\hline $2 p_{3 / 2}$ & & & & & -17.1 & $(4.0)$ \\
\hline $2 p_{1 / 2}$ & & & & & -16.0 & $(2.0)$ \\
\hline $1 g_{9 / 2}$ & & & & & -15.4 & $(2.0)$ \\
\hline $1 g_{7 / 2}$ & & & & & -11.4 & $(2.0)$ \\
\hline $2 d_{5 / 2}$ & & & & & -9.7 & $(0.0)$ \\
\hline $2 d_{3 / 2}$ & & & & & -9.4 & $(0.0)$ \\
\hline $3 s_{1 / 2}$ & & & & & -8.4 & $(0.0)$ \\
\hline $1 h_{11 / 2}$ & & & & & -8.0 & $(0.0)$ \\
\hline
\end{tabular}


TABLE II: Binding energies $\left(B_{\Sigma^{-}}\right)$and widths $\left(\Gamma_{\Sigma}\right)$ of the $\Sigma^{-}$-nucleus $(n \ell)_{\Sigma}$ bound states for $\Sigma^{-}{ }^{27} \mathrm{Al}\left({ }_{\Sigma^{-}}^{28} \mathrm{Mg}\right), \Sigma^{-}-^{57} \mathrm{Co}\left({ }_{\Sigma^{-}}^{58} \mathrm{Fe}\right)$ and $\Sigma^{-} \_207 \mathrm{Tl}\left({ }_{\Sigma^{-}}^{207} \mathrm{Hg}\right)$. Here the $t_{\text {eff }} \rho$ and DD potentials are used as a $\Sigma$-nucleus potential. These values are estimated with the $\Sigma$-nucleus potential plus the finite Coulomb potential (Full) and only the finite Coulomb potential (Coulomb). $k^{\text {(pole) }}$ denotes a corresponding pole position of the bound state in the complex $k$-plane.

\begin{tabular}{|c|c|c|c|c|c|c|c|c|c|c|}
\hline \multirow[b]{2}{*}{$(n \ell)_{\Sigma}$} & \multicolumn{4}{|c|}{$t_{\mathrm{eff}} \rho$ (Full) } & \multicolumn{2}{|c|}{ Coulomb } & \multicolumn{4}{|c|}{ DD (Full) } \\
\hline & $\begin{array}{l}-B_{\Sigma^{-}} \\
(\mathrm{MeV})\end{array}$ & $\begin{array}{c}\Gamma_{\Sigma} \\
(\mathrm{MeV})\end{array}$ & $\begin{array}{c}k^{(\text {pole })} \\
\left(\mathrm{fm}^{-1}\right)\end{array}$ & $\begin{array}{l}\mathrm{rms} \\
(\mathrm{fm})\end{array}$ & $\begin{array}{l}-B_{\Sigma^{-}} \\
(\mathrm{MeV})\end{array}$ & $\begin{array}{l}\mathrm{rms} \\
(\mathrm{fm})\end{array}$ & $\begin{array}{l}-B_{\Sigma^{-}} \\
(\mathrm{MeV})\end{array}$ & $\begin{array}{c}\Gamma_{\Sigma} \\
(\mathrm{MeV})\end{array}$ & $\begin{array}{l}k^{(\text {pole })} \\
\left(\mathrm{fm}^{-1}\right)\end{array}$ & $\begin{array}{l}\mathrm{rms} \\
(\mathrm{fm})\end{array}$ \\
\hline \multicolumn{11}{|l|}{$\Sigma_{\Sigma^{-}}^{28} \mathrm{Mg}$} \\
\hline $1 s$ & -22.0 & 26.9 & $-0.334+i 1.186$ & 2.1 & -2.96 & 5.2 & -1.71 & 1.25 & $-0.057+i 0.322$ & 9.8 \\
\hline $1 p$ & -9.54 & 20.4 & $-0.360+i 0.831$ & 2.9 & -1.25 & 10.4 & -1.13 & 0.49 & $-0.028+i 0.259$ & 12.4 \\
\hline $1 d$ & +2.26 & 10.6 & $-0.486+i 0.322$ & 5.9 & -0.57 & 20.4 & -0.58 & 0.023 & $-0.002+i 0.184$ & 20.2 \\
\hline $2 s$ & +0.26 & 6.12 & $-0.313+i 0.287$ & 11.9 & -0.97 & 15.6 & -0.71 & 0.26 & $-0.019+i 0.205$ & 23.4 \\
\hline $2 p$ & -0.99 & 0.25 & $-0.015+i 0.242$ & 14.1 & -0.56 & 25.1 & -0.53 & 0.015 & $-0.013+i 0.176$ & 28.8 \\
\hline \multicolumn{11}{|l|}{$\stackrel{58}{\Sigma^{-}} \mathrm{Fe}$} \\
\hline $1 s$ & -27.1 & 23.7 & $-0.273+i 1.305$ & 2.5 & -7.13 & 4.2 & -3.89 & 1.89 & $-0.058+i 0.488$ & 9.1 \\
\hline $1 p$ & -19.2 & 21.1 & $-0.286+i 1.111$ & 3.2 & -4.39 & 6.4 & -3.28 & 1.43 & $-0.048+i 0.447$ & 9.8 \\
\hline $1 d$ & -10.6 & 17.7 & $-0.311+i 0.855$ & 3.9 & -2.46 & 10.0 & -2.31 & 0.63 & $-0.026+i 0.373$ & 11.7 \\
\hline $1 f$ & -1.71 & 12.7 & $-0.382+i 0.500$ & 5.4 & -1.42 & 16.2 & -1.43 & 0.080 & $-0.004+i 0.293$ & 16.2 \\
\hline $2 s$ & -9.44 & 15.8 & $-0.294+i 0.809$ & 4.4 & -3.08 & 9.9 & -1.91 & 0.49 & $-0.022+i 0.340$ & 17.9 \\
\hline $2 p$ & -1.74 & 7.23 & $-0.261+i 0.416$ & 11.3 & -2.11 & 13.9 & -1.70 & 0.42 & $-0.020+i 0.320$ & 19.3 \\
\hline \multicolumn{11}{|l|}{${ }_{\Sigma^{-}}^{208} \mathrm{Hg}$} \\
\hline $1 s$ & -38.4 & 19.1 & $-0.190+i 1.543$ & 3.5 & -18.80 & 4.2 & -10.5 & 1.7 & $-0.032+i 0.803$ & 9.8 \\
\hline $1 p$ & -34.2 & 18.6 & $-0.195+i 1.459$ & 4.3 & -15.82 & 5.4 & -10.2 & 1.6 & $-0.031+i 0.788$ & 9.9 \\
\hline $1 d$ & -29.5 & 17.9 & $-0.201+i 1.358$ & 4.8 & -12.92 & 6.5 & -9.4 & 1.4 & $-0.029+i 0.758$ & 10.1 \\
\hline $1 f$ & -24.3 & 16.9 & $-0.209+i 1.236$ & 5.2 & -10.17 & 7.7 & -8.3 & 1.2 & $-0.025+i 0.714$ & 10.4 \\
\hline $2 s$ & -28.5 & 17.4 & $-0.199+i 1.334$ & 4.5 & -13.02 & 6.7 & -6.5 & 0.61 & $-0.015+i 0.633$ & 14.7 \\
\hline $2 p$ & -22.6 & 16.0 & $-0.205+i 1.194$ & 5.0 & -10.50 & 8.2 & -6.4 & 0.58 & $-0.014+i 0.623$ & 14.9 \\
\hline $2 d$ & -16.6 & 14.0 & $-0.208+i 1.029$ & 5.6 & -8.41 & 10.1 & -6.0 & 0.53 & $-0.013+i 0.604$ & 15.3 \\
\hline $3 s$ & -16.0 & 13.1 & $-0.199+i 1.008$ & 5.7 & -8.69 & 10.3 & -4.6 & 0.34 & $-0.010+i 0.530$ & 20.4 \\
\hline $3 p$ & -10.3 & 8.30 & $-0.157+i 0.807$ & 7.3 & -7.17 & 12.4 & -4.5 & 0.32 & $-0.009+i 0.523$ & 20.7 \\
\hline
\end{tabular}


TABLE III: Integrated lab cross sections of $\Sigma^{-}$-nucleus bound states $J^{\pi}$ for $\Sigma^{-}{ }^{57}$ Co with the $t_{\text {eff }} \rho$ potential, by transitions $\left(n \ell_{j}\right)_{p} \rightarrow\left(n \ell_{j}\right)_{\Sigma}$ in the $\left(K^{-}, \pi^{+}\right)$reaction on the ${ }^{58} \mathrm{Ni}$ target at the incident $K^{-}$lab momentum $p_{K}=600 \mathrm{MeV} / \mathrm{c}$ and $\theta_{\text {lab }}=5^{\circ}$. The Fermi-averaged cross section of $\langle d \sigma / d \Omega\rangle_{\text {lab }}^{K^{-} p \rightarrow \pi^{+} \Sigma^{-}}=1.25 \mathrm{mb} / \mathrm{sr}$ and distortion parameters $\sigma_{K}=32 \mathrm{mb}, \sigma_{\pi}=30 \mathrm{mb}$ and $\alpha_{K}=\alpha_{\pi}=0$ are used in the DWIA.

\begin{tabular}{|c|c|c|c|c|c|c|c|c|c|}
\hline \multirow[b]{2}{*}{ Transition } & \multirow[b]{2}{*}{$J^{\pi}$} & \multicolumn{3}{|c|}{$t_{\text {eff }} \rho$ (Real only) } & \multicolumn{5}{|c|}{$t_{\text {eff }} \rho$ (Full) } \\
\hline & & $\begin{array}{l}-B_{\Sigma-} \\
(\mathrm{MeV})\end{array}$ & $\begin{array}{r}\operatorname{Re} P_{\text {eff }} \\
\left(\times 10^{-2}\right)\end{array}$ & $\begin{array}{c}d \sigma / d \Omega \\
(\mu \mathrm{b} / \mathrm{sr})\end{array}$ & $\begin{array}{l}-B_{\Sigma^{-}} \\
(\mathrm{MeV})\end{array}$ & $\begin{array}{r}\Gamma_{\Sigma} \\
(\mathrm{MeV})\end{array}$ & $\begin{array}{r}\operatorname{Re} P_{\text {eff }} \\
\left(\times 10^{-2}\right)\end{array}$ & $\begin{array}{r}\operatorname{Arg} P_{\text {eff }} \\
\text { (deg.) }\end{array}$ & $\begin{array}{l}d \sigma / d \Omega \\
(\mu \mathrm{b} / \mathrm{sr})\end{array}$ \\
\hline \multirow{7}{*}{$\begin{aligned}\left(1 f_{7 / 2}\right)_{p} & \rightarrow\left(1 s_{1 / 2}\right)_{\Sigma} \\
& \rightarrow\left(1 p_{3 / 2}\right)_{\Sigma}\end{aligned}$} & $3^{-}$ & -27.3 & 1.218 & 15.27 & -27.1 & 23.7 & 0.928 & -33.3 & 11.62 \\
\hline & $2^{+}$ & -19.6 & 7.254 & 88.24 & -19.2 & 21.1 & 6.129 & -25.8 & 74.42 \\
\hline & $4^{+}$ & & 0.873 & 10.62 & & & 0.665 & -34.5 & 8.07 \\
\hline & $4^{+}$ & -19.6 & 1.222 & 14.87 & -19.2 & 21.1 & 0.930 & -34.5 & 11.30 \\
\hline & $1^{-}$ & -11.5 & 17.291 & 203.67 & -10.6 & 17.7 & 16.515 & -17.6 & 193.84 \\
\hline & $3^{-}$ & & 6.790 & 79.98 & & & 5.808 & -28.5 & 68.17 \\
\hline & $5^{-}$ & & 0.599 & 7.05 & & & 0.502 & -31.5 & 5.89 \\
\hline \multirow[t]{2}{*}{$\rightarrow\left(1 d_{3 / 2}\right)_{\Sigma}$} & $3^{-}$ & -11.5 & 2.263 & 26.66 & -10.6 & 17.7 & 1.936 & -28.5 & 22.72 \\
\hline & $5^{-}$ & & 1.397 & 16.45 & & & 1.171 & -31.5 & 13.74 \\
\hline \multirow{4}{*}{$\rightarrow\left(1 f_{7 / 2}\right)_{\Sigma}$} & $0^{+}$ & -3.58 & 3.136 & 35.79 & -1.72 & 12.7 & 4.563 & 30.2 & 51.68 \\
\hline & $2^{+}$ & & 12.780 & 145.86 & & & 15.448 & -13.8 & 174.99 \\
\hline & $4^{+}$ & & 5.146 & 58.73 & & & 5.300 & -29.1 & 60.04 \\
\hline & $6^{+}$ & & 0.235 & 2.68 & & & 0.282 & -23.2 & 3.20 \\
\hline \multirow[t]{3}{*}{$\rightarrow\left(1 f_{5 / 2}\right)_{\Sigma}$} & $2^{+}$ & -3.58 & 1.534 & 17.50 & -1.72 & 12.7 & 1.854 & -13.8 & 21.00 \\
\hline & $4^{+}$ & & 2.859 & 32.63 & & & 2.945 & -29.1 & 33.36 \\
\hline & $6^{+}$ & & 0.704 & 8.03 & & & 0.847 & -23.2 & 9.60 \\
\hline
\end{tabular}


TABLE IV: Integrated lab cross sections of $\Sigma^{-}$-nucleus bound states $J^{\pi}$ for $\Sigma^{-}{ }^{57}$ Co with the DD potential, by transitions $\left(n \ell_{j}\right)_{p} \rightarrow\left(n \ell_{j}\right)_{\Sigma}$ in the $\left(K^{-}, \pi^{+}\right)$reaction on the ${ }^{58} \mathrm{Ni}$ target at the incident $K^{-}$lab momentum $p_{K}=600 \mathrm{MeV} / \mathrm{c}\left(5^{\circ}\right)$. See the caption in Table III.

\begin{tabular}{|c|c|c|c|c|c|c|c|c|c|}
\hline \multirow[b]{2}{*}{ Transition } & \multirow[b]{2}{*}{$J^{\pi}$} & \multicolumn{3}{|c|}{ DD (Real only) } & \multicolumn{5}{|c|}{ DD (Full) } \\
\hline & & $\begin{array}{l}-B_{\Sigma^{-}} \\
(\mathrm{MeV})\end{array}$ & $\begin{array}{r}\operatorname{Re} P_{\text {eff }} \\
\left(\times 10^{-2}\right)\end{array}$ & $\begin{array}{l}d \sigma / d \Omega \\
(\mu \mathrm{b} / \mathrm{sr})\end{array}$ & $\begin{array}{l}-B_{\Sigma^{-}} \\
(\mathrm{MeV})\end{array}$ & $\begin{array}{r}\Gamma_{\Sigma} \\
(\mathrm{MeV})\end{array}$ & $\begin{array}{r}\operatorname{Re} P_{\text {eff }} \\
\left(\times 10^{-2}\right)\end{array}$ & $\begin{array}{r}\operatorname{Arg} P_{\text {eff }} \\
\quad(\text { deg. })\end{array}$ & $\begin{array}{l}d \sigma / d \Omega \\
(\mu \mathrm{b} / \mathrm{sr})\end{array}$ \\
\hline \multirow{7}{*}{$\begin{aligned}\left(1 f_{7 / 2}\right)_{p} & \rightarrow\left(1 s_{1 / 2}\right)_{\Sigma} \\
& \rightarrow\left(1 p_{3 / 2}\right)_{\Sigma}\end{aligned}$} & $3^{-}$ & -4.46 & 5.147 & 58.95 & -3.89 & 1.89 & 3.539 & 45.2 & 40.45 \\
\hline & $2^{+}$ & -3.74 & 4.391 & 50.15 & -3.28 & 1.43 & 2.265 & 55.6 & 25.82 \\
\hline & $4^{+}$ & & 1.895 & 21.65 & & & 1.260 & 45.9 & 14.36 \\
\hline & $4^{+}$ & -3.74 & 2.653 & 30.30 & -3.28 & 1.43 & 1.764 & 45.9 & 20.11 \\
\hline & $1^{-}$ & -2.55 & 1.979 & 22.49 & -2.31 & 0.63 & 0.386 & 75.0 & 4.38 \\
\hline & $3^{-}$ & & 2.366 & 26.89 & & & 0.888 & 61.6 & 10.09 \\
\hline & $5^{-}$ & & 0.366 & 4.16 & & & 0.167 & 55.8 & 1.90 \\
\hline \multirow[t]{2}{*}{$\rightarrow\left(1 d_{3 / 2}\right)_{\Sigma}$} & $3^{-}$ & -2.55 & 0.789 & 8.96 & -2.31 & 0.63 & 0.296 & 61.6 & 3.36 \\
\hline & $5^{-}$ & & 0.854 & 9.71 & & & 0.390 & 55.8 & 4.42 \\
\hline \multirow[t]{4}{*}{$\rightarrow\left(1 f_{7 / 2}\right)_{\Sigma}$} & $0^{+}$ & -1.46 & 0.008 & 0.09 & -1.43 & 0.080 & 0.010 & -6.9 & 0.12 \\
\hline & $2^{+}$ & & 0.288 & 3.25 & & & 0.111 & 56.5 & 1.26 \\
\hline & $4^{+}$ & & 0.258 & 2.92 & & & 0.126 & 47.8 & 1.42 \\
\hline & $6^{+}$ & & 0.010 & 0.12 & & & 0.005 & 49.5 & 0.05 \\
\hline \multirow[t]{3}{*}{$\rightarrow\left(1 f_{5 / 2}\right)_{\Sigma}$} & $2^{+}$ & -1.46 & 0.035 & 0.39 & -1.43 & 0.080 & 0.013 & 56.5 & 0.15 \\
\hline & $4^{+}$ & & 0.143 & 1.62 & & & 0.070 & 47.8 & 0.79 \\
\hline & $6^{+}$ & & 0.031 & 0.35 & & & 0.014 & 49.5 & 0.16 \\
\hline
\end{tabular}




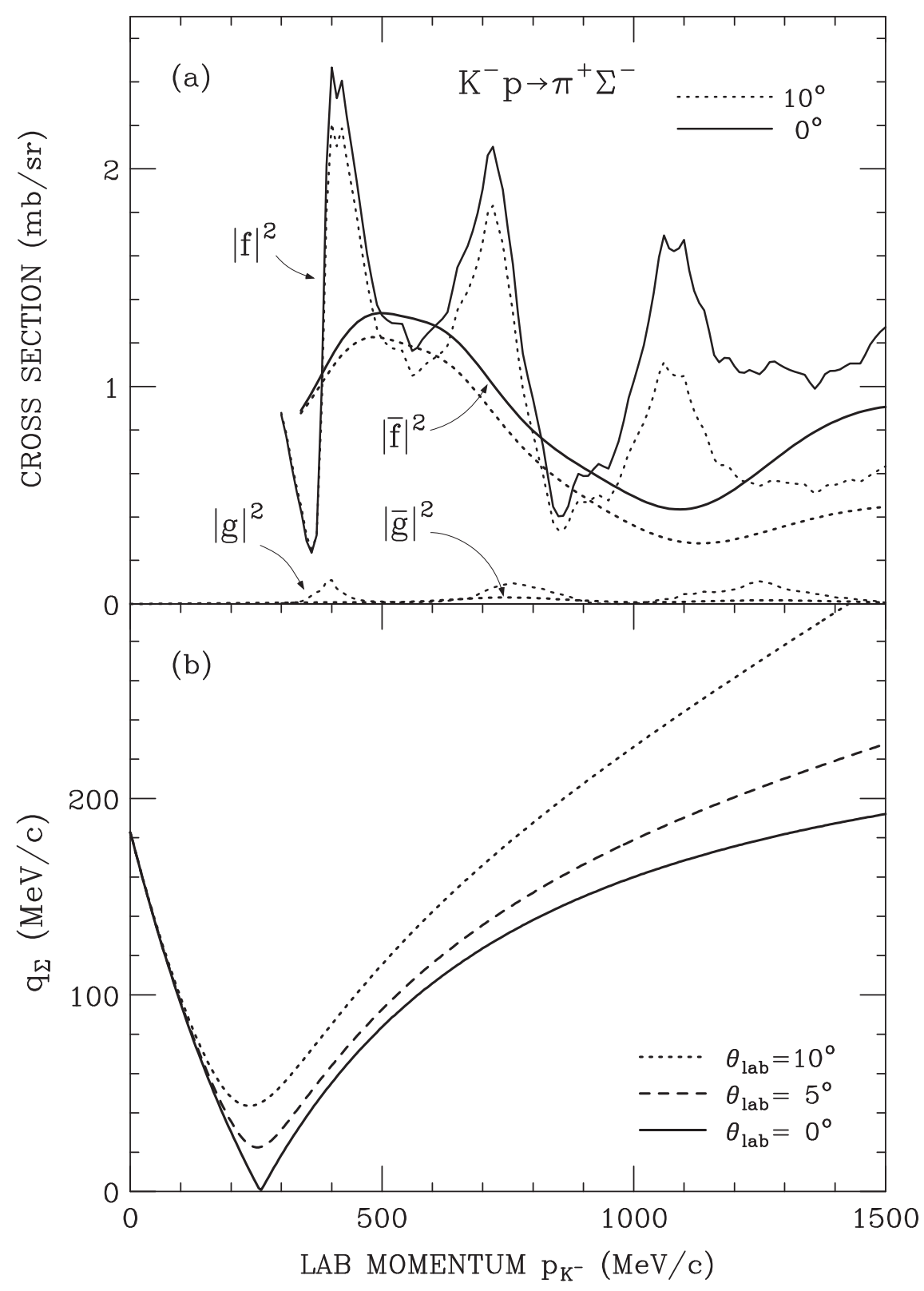

FIG. 1: (a) Absolute cross sections of non-spin-flip and spin-flip processes, $|f|^{2}$ and $|g|^{2}$, for the $K^{-}+p \rightarrow \pi^{-}+\Sigma^{-}$reaction in free space [33], as a function of the incident $K^{-}$lab momentum $p_{K}$, together with the Fermi-averaged cross sections, $|\bar{f}|^{2}$ and $|\bar{g}|^{2}$, in nuclear medium. The solid and dotted curves denote the values at the detected $\pi^{+}$angle $\theta_{\text {lab }}=0^{\circ}$ and $10^{\circ}$, respectively. (b) Momentum transfer $q_{\Sigma}$ for a $\Sigma^{-}$production by the $\left(K^{-}, \pi^{+}\right)$reaction on a ${ }^{58} \mathrm{Ni}$ target, as a function of the incident $K^{-}$lab momentum $p_{K}$. The solid, dashed and dotted curves denote for $\theta_{\text {lab }}=0^{\circ}, 5^{\circ}$ and $10^{\circ}$ in the lab frame, respectively. 


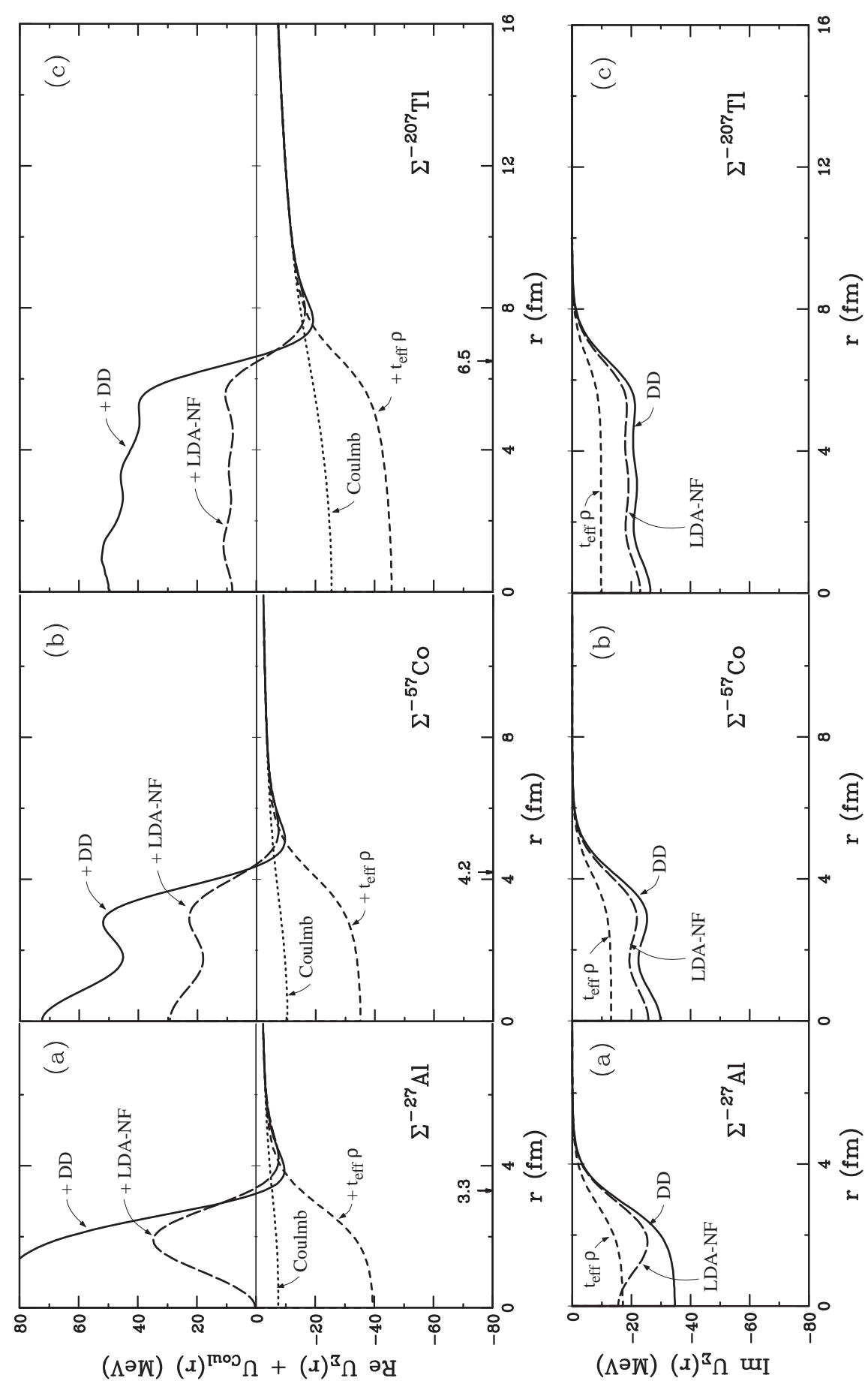

FIG. 2: (top) Real and (bottom) imaginary parts of the $\Sigma$-nucleus potential plus the finite Coulomb potential for (a) $\Sigma^{-} \_27 \mathrm{Al}$, (b) $\Sigma^{-} \_57 \mathrm{Co}$ and (c) $\Sigma^{-} \_207 \mathrm{Tl}$. The solid, long-dashed and dashed curves denote the radial distribution of the potentials for DD, LDA-NF and $t_{\text {eff }} \rho$, respectively. The strength for the real part includes the finite Coulomb potential. The dotted curves denote only the Coulomb potential for the $\Sigma^{-}$-nucleus systems. 


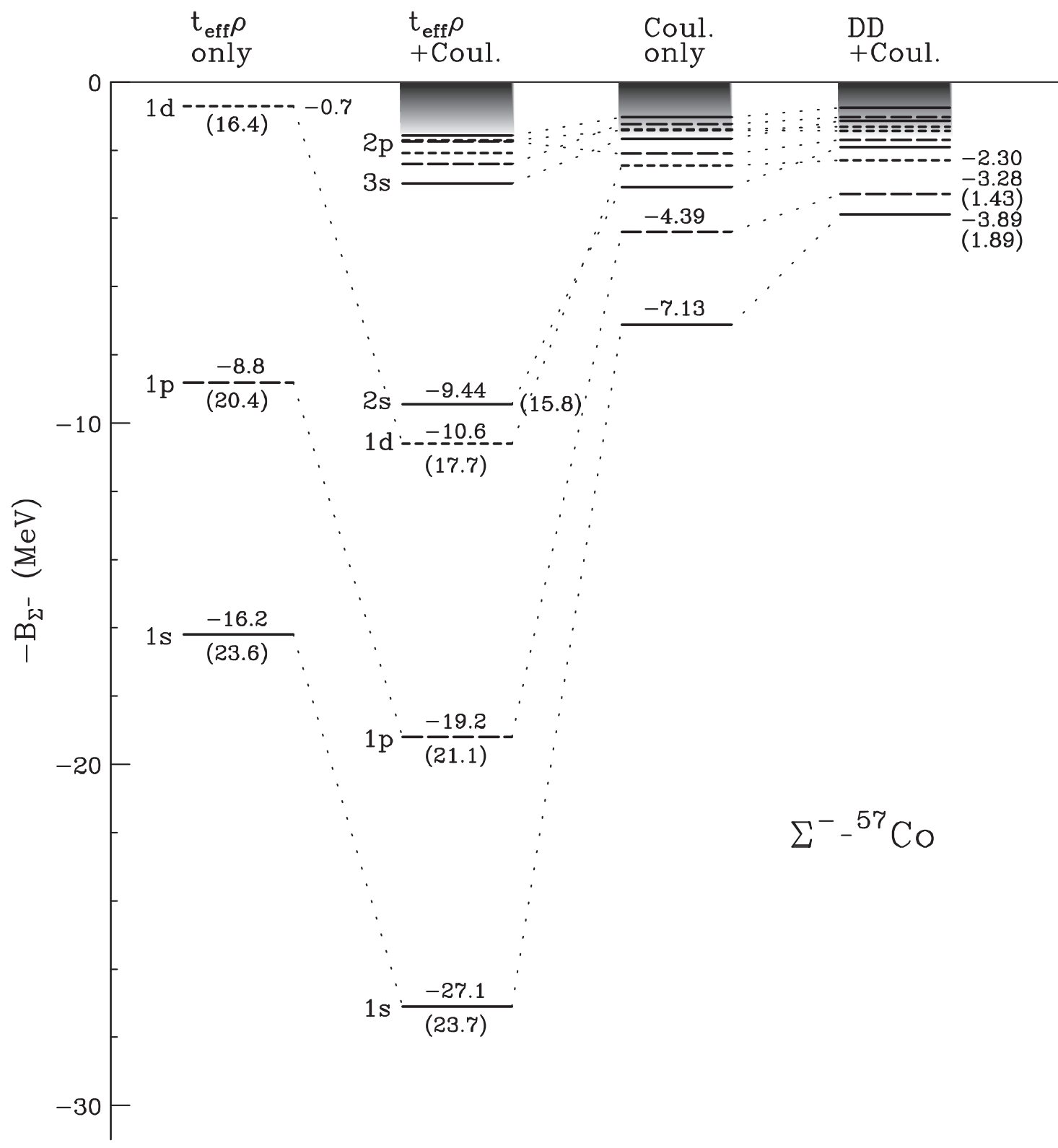

FIG. 3: Binding energies and widths (in the brackets) of the $\Sigma^{-}(n s)_{\Sigma},(n p)_{\Sigma}$ and $(n d)_{\Sigma}$ bound states in $\Sigma^{-}{ }^{57}$ Co. These values are obtained by the $t_{\text {eff }} \rho$ potential only, the $t_{\text {eff }} \rho$ or DD potential including the finite Coulomb potential, and the finite Coulomb potential only. The $\Sigma^{-}$atomic states occur in the shaded region. See Table II. 




FIG. 4: Density distributions of $\Sigma^{-}$-nucleus $1 s, 2 s, 1 p, 2 p, 1 d$ and $1 f$ bound states in $\Sigma^{-}-^{27} \mathrm{Al}$ (left), $\Sigma^{-}{ }^{57} \mathrm{Co}$ (middle) and $\Sigma^{-} \_207 \mathrm{Tl}$ (right) systems. The solid and dashed curves denote the density distributions of $r^{2} \rho_{n \ell}(r)$ for the DD and LDA-NF potentials, respectively, taken into account the finite Coulomb potential. The dotted curves denote the density distributions of the finite Coulomb bound states. 


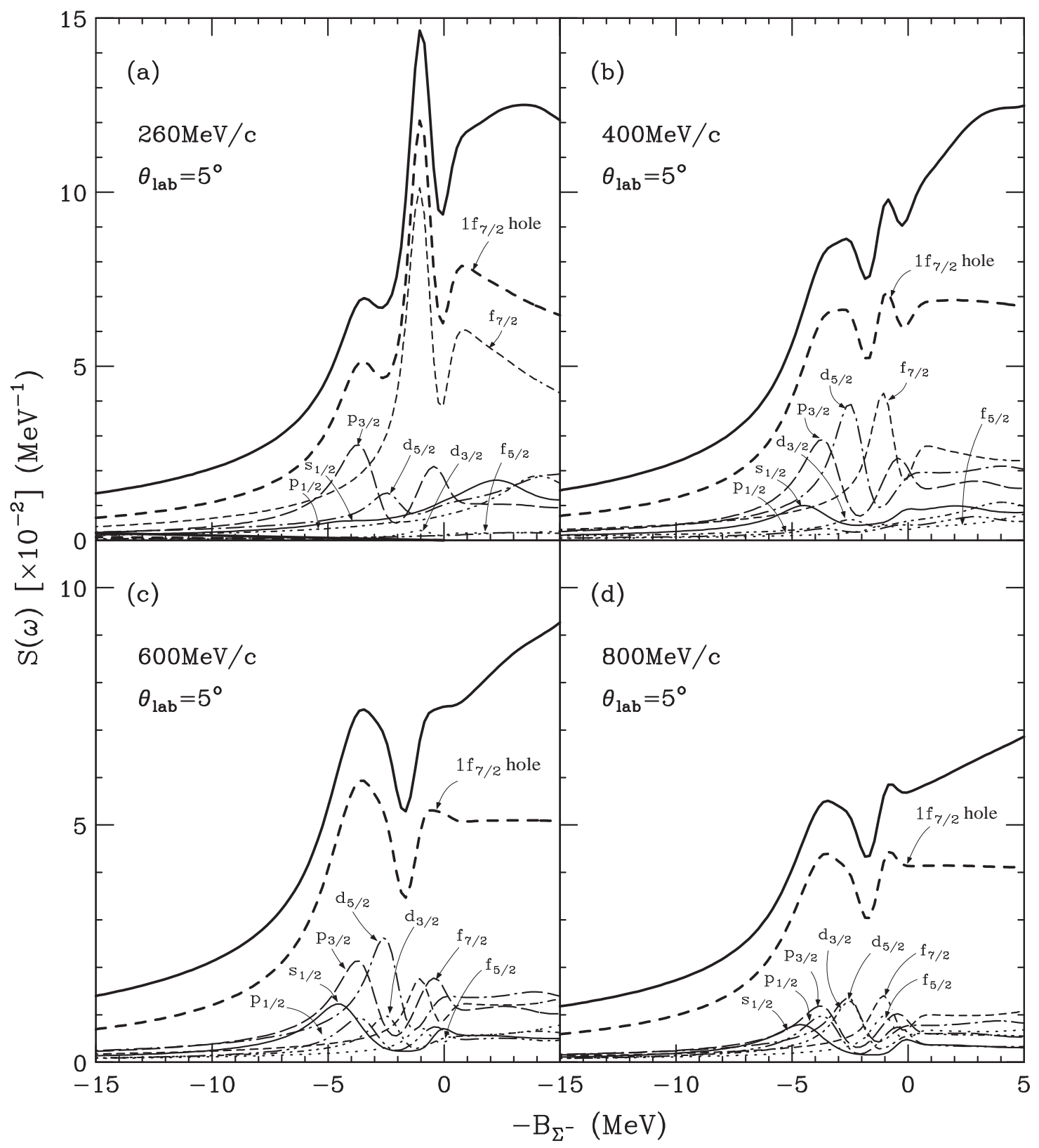

FIG. 5: Partial-wave components of the calculated strength function $S(\omega)$ for the inclusive spectra

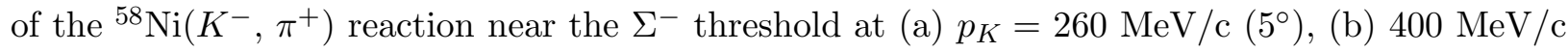
$\left(5^{\circ}\right)$, (c) $600 \mathrm{MeV} / \mathrm{c}\left(5^{\circ}\right)$ and (d) $800 \mathrm{MeV} / \mathrm{c}\left(5^{\circ}\right)$, as a function of a $\Sigma^{-}$binding energy $-B_{\Sigma^{-}}$. Here the DD potential is used. The thick solid and thick dashed curves denote a total inclusive spectrum and a total contribution of a $1 f_{7 / 2}^{-1}$ proton-hole state in the ${ }^{58} \mathrm{Ni}$ target. The spectra are folded with a detector resolution of $1.5 \mathrm{MeV}$ FWHM. 


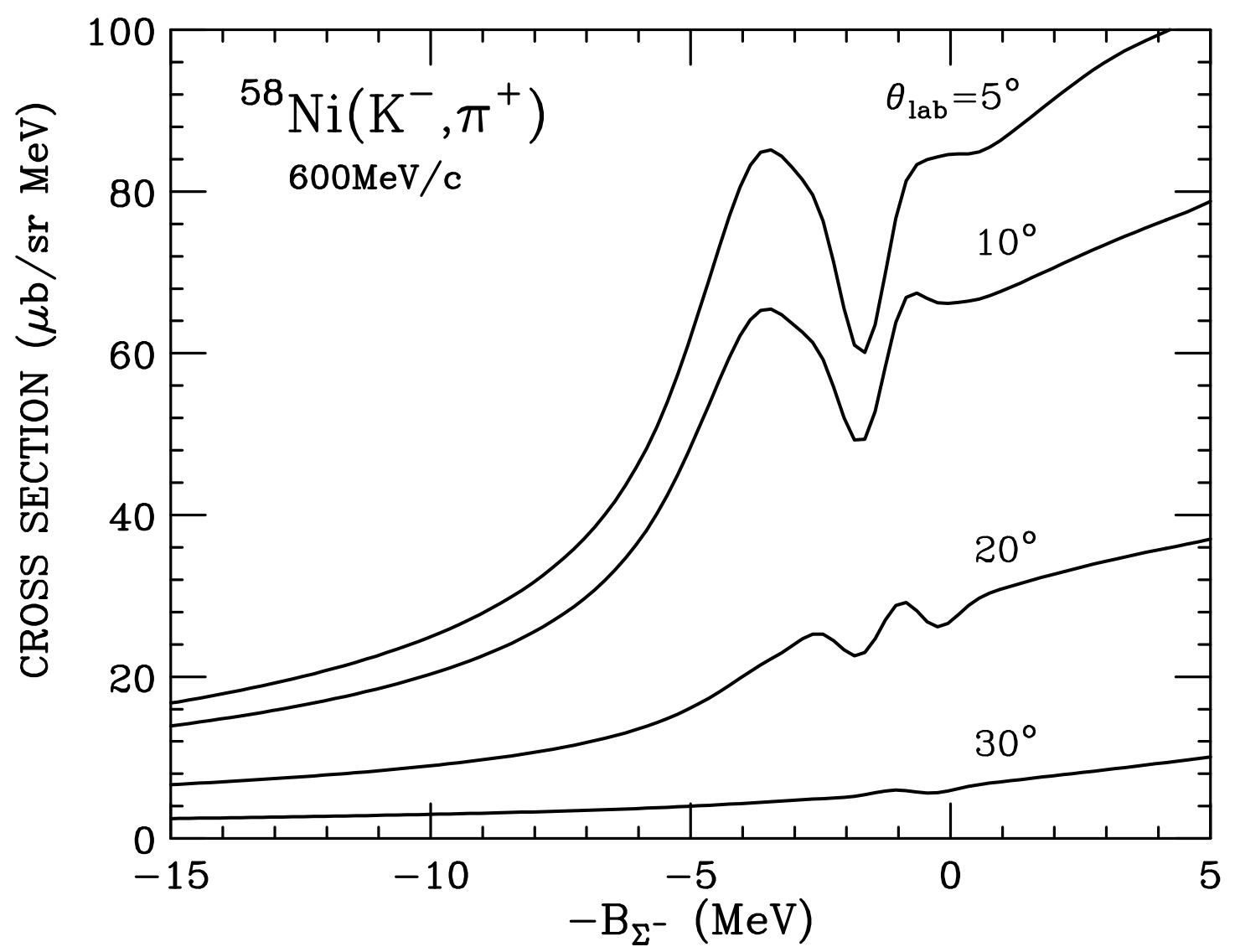

FIG. 6: Angular dependence of the calculated inclusive spectra of the ${ }^{58} \mathrm{Ni}\left(K^{-}, \pi^{+}\right)$reaction at $p_{K}=600 \mathrm{MeV} / \mathrm{c}$ near the $\Sigma^{-}$threshold, as a function of $-B_{\Sigma^{-}}$. The curves denote the spectra for $\theta_{\text {lab }}=5^{\circ}, 10^{\circ}, 20^{\circ}$ and $30^{\circ}$, respectively. The DD potential is used. The spectra are folded with a detector resolution of $1.5 \mathrm{MeV}$ FWHM. 


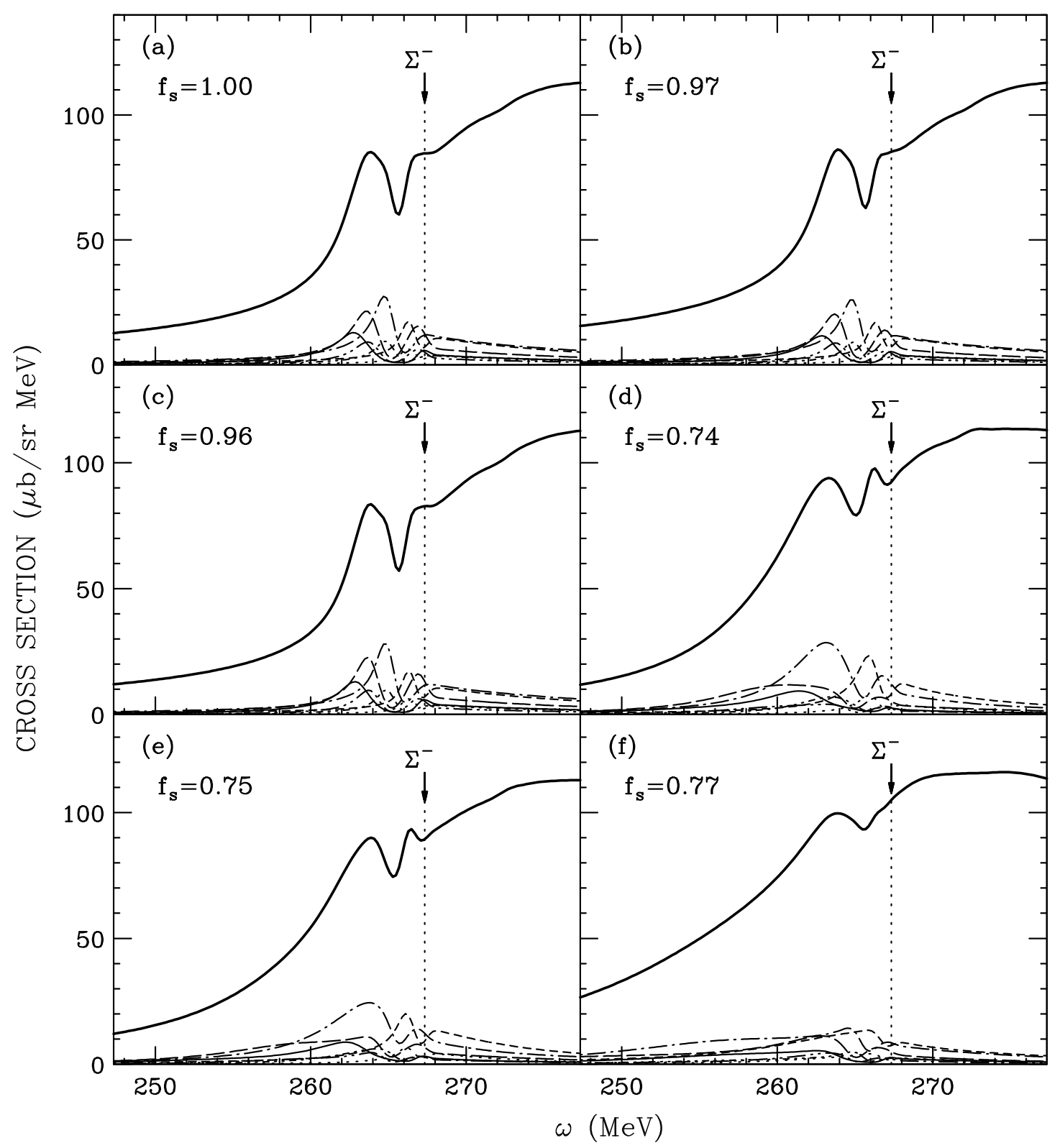

FIG. 7: Calculated inclusive spectra of the ${ }^{58} \mathrm{Ni}\left(K^{-}, \pi^{+}\right)$reactions at $p_{K}=600 \mathrm{MeV} / \mathrm{c}\left(5^{\circ}\right)$ near the $\Sigma^{-}$threshold. The thick solid curve denotes the spectra with the potentials of (a) DD, (b) RMF, (c) LDA-NF, (d) LDA-S3, (e) WS-sh and (f) $t_{\text {eff }} \rho$, where each calculated spectrum is normalized by a factor $f_{s}$. The partial-wave contributions to the inclusive spectra are also drawn (see the Fig. 5(c)). The spectra are folded with a detector resolution of $1.5 \mathrm{MeV}$ FWHM. The arrows show the $\Sigma^{-}+{ }^{57}$ Co threshold energy at $\omega=267.33 \mathrm{MeV}$. 


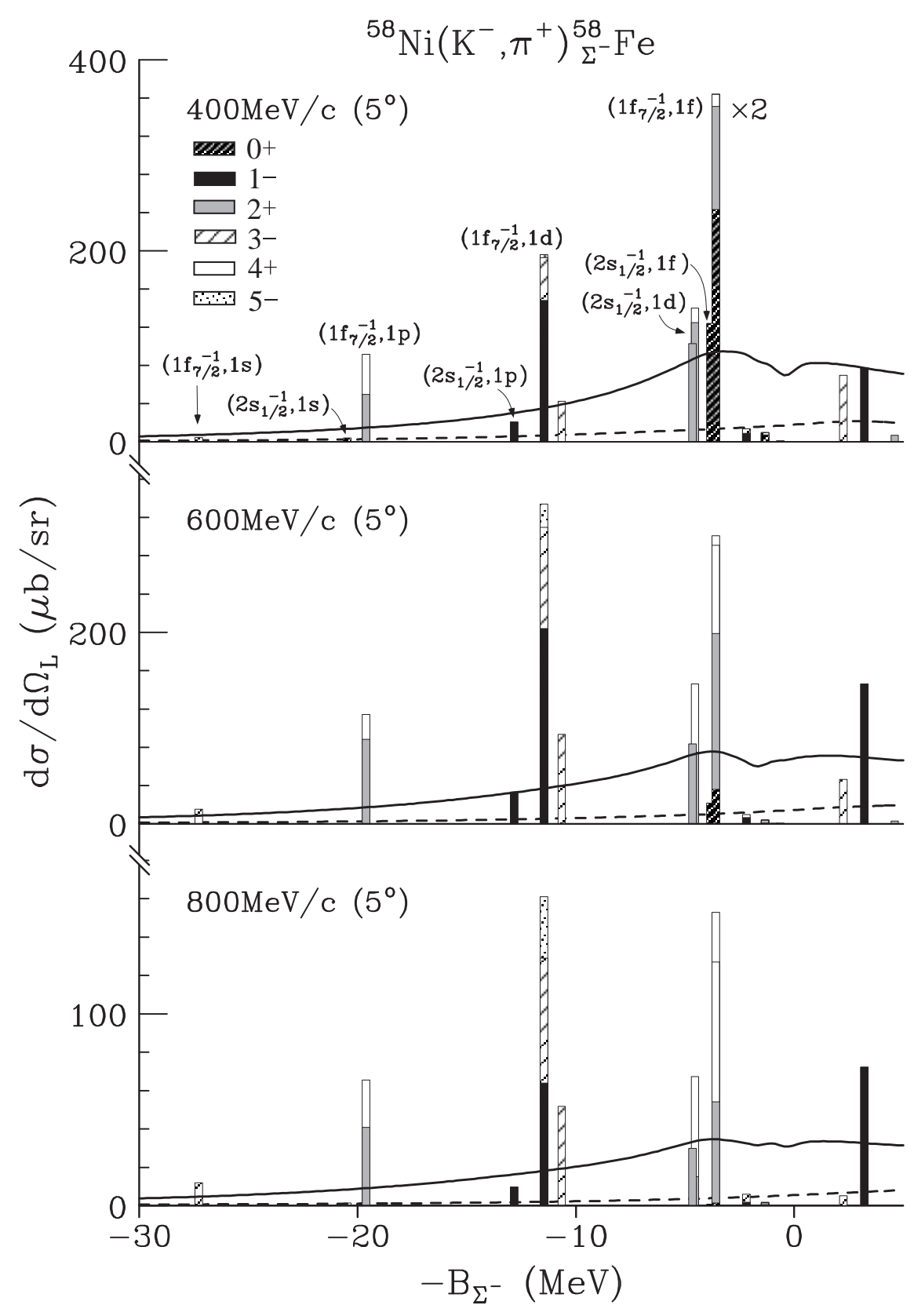

FIG. 8: Integrated cross sections of the ${ }^{58} \mathrm{Ni}\left(K^{-}, \pi^{+}\right)$reactions at $p_{K}=400,600$ and $800 \mathrm{MeV} / \mathrm{c}$ $\left(5^{\circ}\right)$ with the $t_{\text {eff }} \rho$ potential. The values are calculated by the real part of the $\Sigma$-nucleus potential including the finite Coulomb one, and its imaginary part is omitted. The solid and dashed curves denote the contributions of the spectra from proton-hole states of $1 f_{7 / 2}^{-1}$ (solid) and $2 s_{1 / 2}^{-1}$ (dash) in the ${ }^{58} \mathrm{Ni}$ target when the imaginary part of the potential is switched on. 


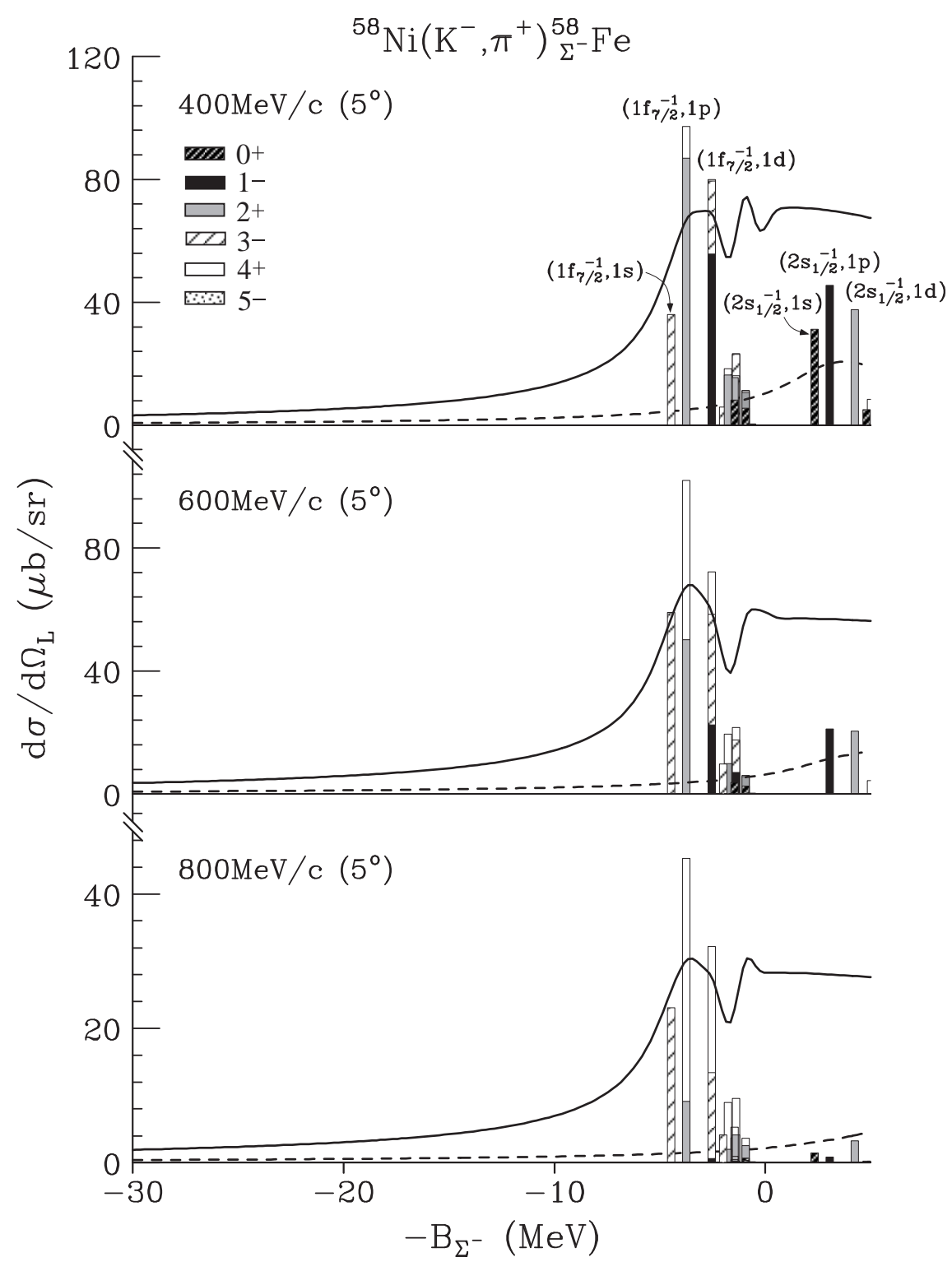

FIG. 9: Integrated cross sections of the ${ }^{58} \mathrm{Ni}\left(K^{-}, \pi^{+}\right)$reactions at $p_{K}=400,600$ and $800 \mathrm{MeV} / \mathrm{c}$ $\left(5^{\circ}\right)$ with the DD potential. See the caption in Fig. 8. 


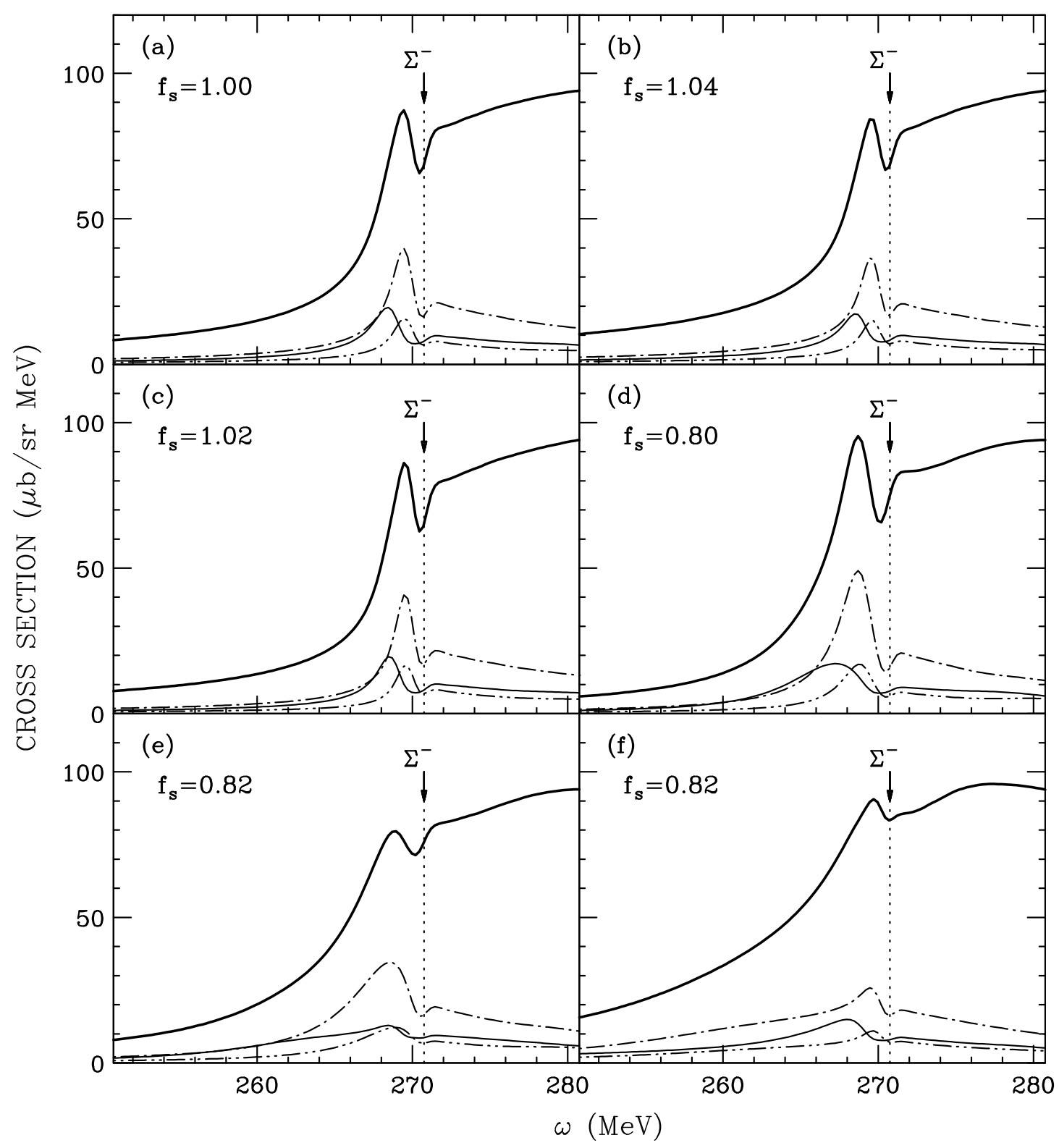

FIG. 10: Calculated inclusive spectra of the ${ }^{28} \mathrm{Si}\left(K^{-}, \pi^{+}\right)$reactions at $p_{K}=600 \mathrm{MeV} / \mathrm{c}\left(5^{\circ}\right)$ near the $\Sigma^{-}$threshold. The thick solid curve denotes the spectra with the potentials of (a) DD, (b) RMF, (c) LDA-NF, (d) LDA-S3, (e) WS-sh and (f) $t_{\mathrm{eff}} \rho$, where each calculated spectrum is normalized by a factor $f_{s}$. The solid, dot-dashed and dot-dot-dashed curves denote the partial-wave contributions of $\left(s_{1 / 2}\right)_{\Sigma},\left(p_{3 / 2,1 / 2}\right)_{\Sigma}$ and $\left(d_{5 / 2,3 / 2}\right)_{\Sigma}$ states for $\Sigma^{-}-^{27} \mathrm{Al}$, respectively. The spectra are folded with a detector resolution of $1.5 \mathrm{MeV}$ FWHM. The arrows show the $\Sigma^{-}{ }^{27} \mathrm{Al}$ threshold energy at $\omega=270.75 \mathrm{MeV}$. 


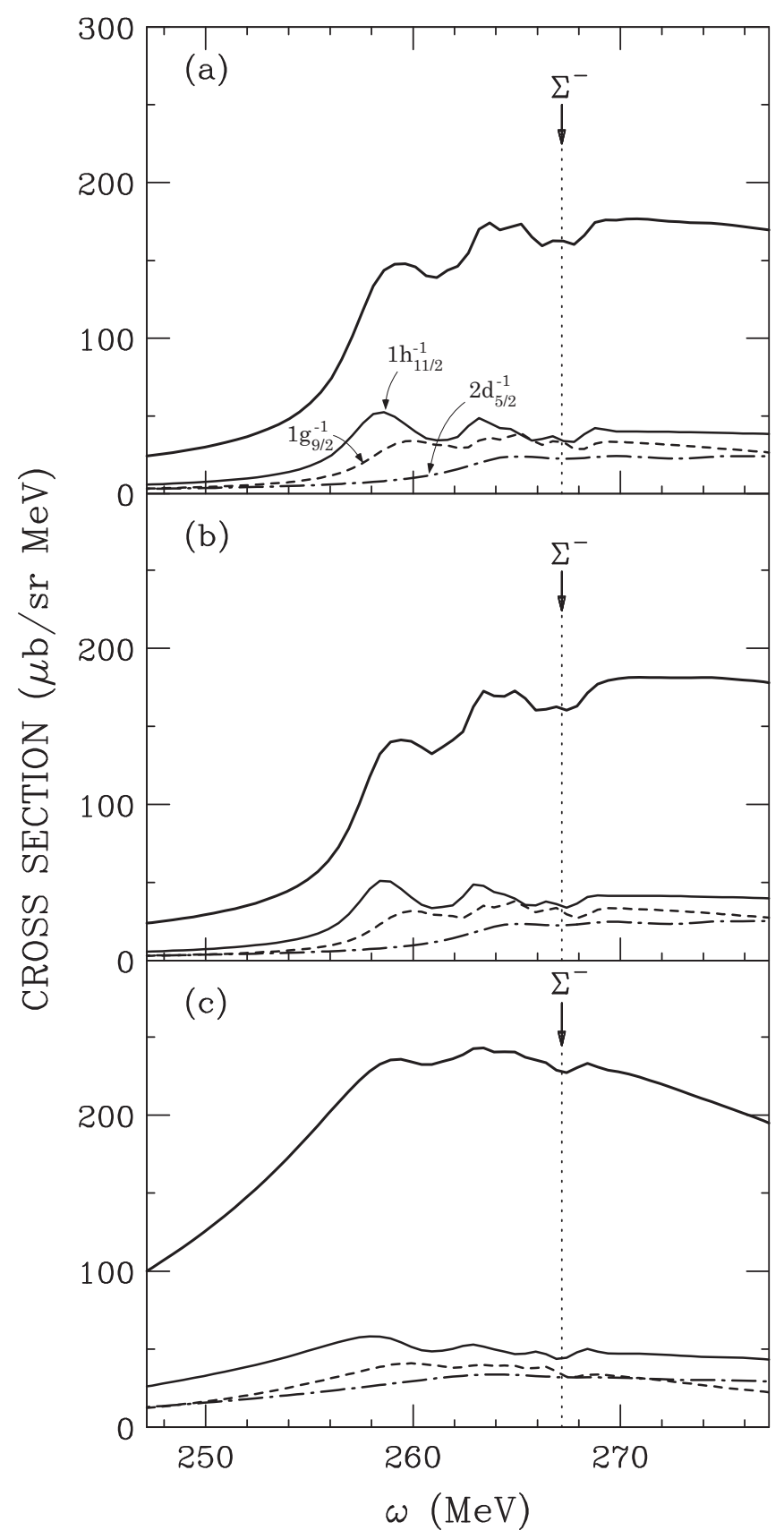

FIG. 11: Calculated spectra with 16 proton-hole states involving $1 h_{11 / 2}^{-1}, 2 d_{5 / 2,3 / 2}^{-1}$ and $1 g_{9 / 2,7 / 2}^{-1}$ in the ${ }^{208} \mathrm{~Pb}\left(K^{-}, \pi^{+}\right)$reaction at $p_{K}=600 \mathrm{MeV} / \mathrm{c}\left(5^{\circ}\right)$ near the $\Sigma^{-}$threshold, with the (a) DD, (b) LDA-NF and (c) $t_{\text {eff }} \rho$ potentials. The thick solid curves denote the total spectra with each potential. The solid, dash and dot-dashed curves denote the contributions of the $1 h_{11 / 2}^{-1}, 2 d_{5 / 2}^{-1}$ and $1 g_{9 / 2}^{-1}$ components, respectively. The spectra are folded with a detector resolution of $1.5 \mathrm{MeV}$ FWHM. The arrows show the $\Sigma^{-}+{ }^{207} \mathrm{Tl}$ threshold energy at $\omega=267.17 \mathrm{MeV}$. 


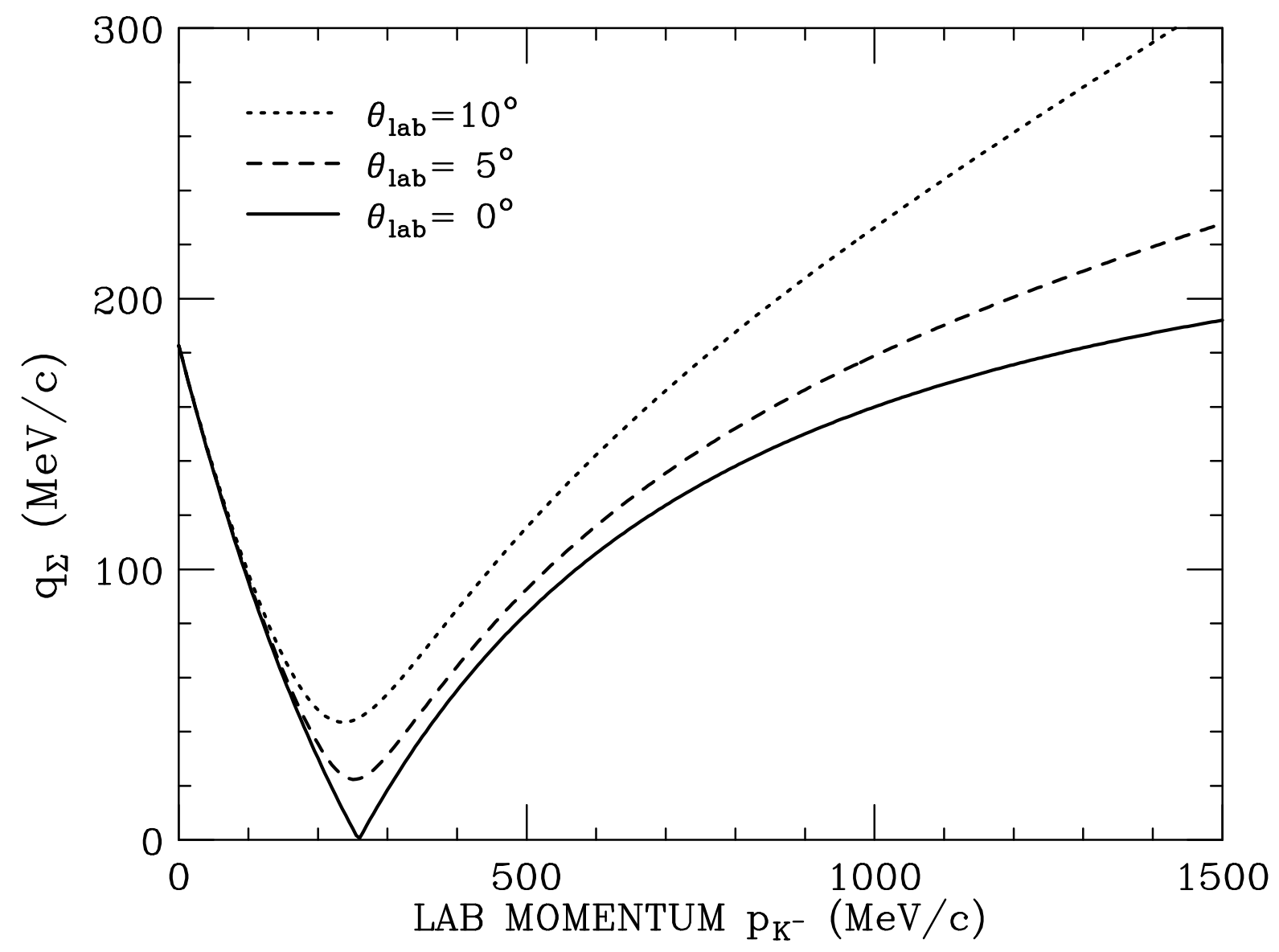

